\title{
Multiple Symmetric Results for Quasilinear Elliptic Systems Involving Singular Potentials and Critical Sobolev Exponents in $\mathbb{R}^{N}$
}

\author{
Zhiying Deng ${ }^{1}$ and Yisheng Huang ${ }^{2}$ \\ ${ }^{1}$ School of Mathematics and Physics, Chongqing University of Posts and Telecommunications, Chongqing 400065, China \\ ${ }^{2}$ Department of Mathematics, Soochow University, Suzhou, Jiangsu 215006, China
}

Correspondence should be addressed to Zhiying Deng; dengzy@cqupt.edu.cn

Received 2 April 2014; Accepted 28 August 2014; Published 16 November 2014

Academic Editor: Márcia Federson

Copyright (C) 2014 Z. Deng and Y. Huang. This is an open access article distributed under the Creative Commons Attribution License, which permits unrestricted use, distribution, and reproduction in any medium, provided the original work is properly cited.

This paper deals with a class of quasilinear elliptic systems involving singular potentials and critical Sobolev exponents in $\mathbb{R}^{N}$. By using the symmetric criticality principle of Palais and variational methods, we prove several existence and multiplicity results of $G$-symmetric solutions under certain appropriate hypotheses on the potentials and parameters.

\section{Introduction}

In this work, we investigate the existence and multiplicity of nontrivial solutions for the following quasilinear elliptic system:

$$
\begin{aligned}
\mathscr{L}_{p, \mu} u= & Q(x)\left(|u|^{p^{*}-2} u+\frac{\varsigma \alpha}{p^{*}}|u|^{\alpha-2} u|v|^{\beta}\right) \\
& +\lambda h_{1}(x)|u|^{q-2} u, \quad \text { in } \mathbb{R}^{N}, \\
\mathscr{L}_{p, \mu} v= & Q(x)\left(|v|^{p^{*}-2} v+\frac{\varsigma \beta}{p^{*}}|u|^{\alpha}|v|^{\beta-2} v\right) \\
& +\lambda h_{2}(x)|v|^{q-2} v, \quad \text { in } \mathbb{R}^{N}, \\
u(x), & v(x) \longrightarrow 0, \quad \text { as }|x| \longrightarrow+\infty,
\end{aligned}
$$

where $\mathscr{L}_{p, \mu} \triangleq-\operatorname{div}\left(|\nabla \cdot|^{p-2} \nabla \cdot\right)-\mu\left(|\cdot|^{p-2} \cdot /|x|^{p}\right)$ is a quasilinear elliptic operator, $1<q<p<N, 0<\varsigma<+\infty, 0 \leq \mu<\bar{\mu}$ with $\bar{\mu}=((N-p) / p)^{p}, \lambda \geq 0$, and $\alpha, \beta>1$ satisfy $\alpha+\beta=p^{*}$, $p^{*} \triangleq(N p /(N-p))$ denotes the critical Sobolev exponent, and $Q \in \mathscr{C}\left(\mathbb{R}^{N}\right) \cap L^{\infty}\left(\mathbb{R}^{N}\right)$ and $h_{i} \in L^{\theta}\left(\mathbb{R}^{N}\right)(i=1,2)$ with $\theta=N p /(N p-q(N-p))$ are $G$-symmetric functions (see Section 2 for details) with respect to a closed subgroup $G$ of $O(\mathbb{N})$.
In recent years, considerable attention has been paid to the scalar singular elliptic problem:

$$
\begin{gathered}
\mathscr{L}_{p, \mu} u=Q(x)|u|^{p^{*}-2} u+\lambda h(x)|u|^{q-2} u, \quad \text { in } \Omega, \\
u=0, \quad \text { on } \partial \Omega,
\end{gathered}
$$

where $\Omega \subset \mathbb{R}^{N}$ is a smooth domain (bounded or unbounded) containing the origin. The study of this type of equation is motivated by its definite physics background and various applications, including celestial mechanics, fluid mechanics, and flow through porous media (see [1]). The mathematical interest lies in the fact that these problems like (2) are doubly critical due to the presence of the Sobolev embedding and the singularities. For this reason, many existence, nonexistence, and multiplicity results of nontrivial solutions for the single equations like (2) have been established with different assumptions on the potentials $Q(x), h(x)$ and the parameters $\mu, \lambda$, and $q$; we refer to [2-8] and the references therein.

In a recent paper, Deng and Jin [9] considered the following single semilinear elliptic problem:

$$
-\Delta u-\mu \frac{u}{|x|^{2}}=\frac{k(x)}{|x|^{s}} u^{2^{*}(s)-1}, \quad u>0 \quad \text { in } \mathbb{R}^{N},
$$


where $N>2,0 \leq \mu<((N-2) / 2)^{2}, 0 \leq s<2$, $2^{*}(s)=2(N-s) /(N-2)$, and $k$ satisfies some symmetry conditions with respect to $G \subset O(\mathbb{N})$. By using analytic techniques and variational arguments, the authors proved the existence and multiplicity of $G$-symmetric solutions to (3) under certain hypotheses on $k$. Subsequently, Waliullah [10] improved the results in [9] by using the minimizing sequence and the concentration-compactness principle. Recently, Deng and Huang [11] extended the results in $[9,10]$ to the scalar weighted elliptic problems in a bounded $G$ symmetric domain. Besides these, when $\mu=s=0$ and the right-hand side term $|x|^{-s} u^{2^{*}(s)-1}$ is replaced by a term $f(u)$ of the pure power, such as $f(u)=u^{r-1}$ with $1<r<2 N /(N-2)$ or $r=2 N /(N-2)$, there are many interesting results on the existence and multiplicity of $G$-symmetric solutions of (3), which can be found in [12-14] and the references therein.

On the other hand, there have been many papers concerned with the existence and multiplicity of nontrivial solutions for elliptic systems. In [15], Wu considered the following semilinear elliptic system:

$$
\begin{gathered}
-\Delta u=\frac{2 \alpha}{\alpha+\beta} Q(x)|u|^{\alpha-2} u|v|^{\beta}+\lambda f(x)|u|^{q-2} u, \quad \text { in } \Omega, \\
-\Delta v=\frac{2 \beta}{\alpha+\beta} Q(x)|u|^{\alpha}|v|^{\beta-2} v+\delta h(x)|v|^{q-2} v, \quad \text { in } \Omega, \\
u=v=0, \quad \text { on } \partial \Omega,
\end{gathered}
$$

where $\Omega \subset \mathbb{R}^{N}(N \geq 3)$ is a smooth bounded domain, $1<q<2, \alpha, \beta>1, \alpha+\beta<2^{*}$, and the weight functions Q, $f, h$ fulfill certain suitable conditions. Via the analytic techniques of Nehari manifold and variational methods, the author proved that the system (4) admits at least two nontrivial nonnegative solutions if the pair of parameters $(\lambda, \delta)$ belongs to a certain subset of $\mathbb{R}^{2}$. Very recently, Nyamoradi [16], Lü and Xiao [17], and Li and Gao [18] generalized the corresponding results of [15] to the nonlinear singular elliptic systems involving critical Hardy-Sobolev exponents. Other results about existence and multiplicity of nontrivial solutions, also for related elliptic systems, can be seen in [1923 ] and the references therein.

However, as far as we know, the existence and multiplicity of $G$-symmetric solutions for singular elliptic systems were seldom studied; we can only find some $G$-symmetric results for singular elliptic systems in [24] and, when $G=O(\mathbb{N})$, some radial and nonradial results for nonsingular elliptic systems in [25]. Inspired by $[9,12,25]$, in this paper we are concerned with the existence and multiplicity of positive $G$ symmetric solutions for system (1). The main difficulties lie in the fact that there are not only the nonlinear perturbations $\lambda h_{1}(x)|u|^{q-2} u, \lambda h_{2}(x)|v|^{q-2} v$ and the Hardy singular terms $|u|^{p-2} u /|x|^{p},|v|^{p-2} v /|x|^{p}$ in (1), but also four nonlinear terms with the critical Sobolev exponents in $\mathbb{R}^{N}$. Compared with (3) and (4), the singular quasilinear elliptic system (1) becomes more complicated to deal with. Moreover, the approach involving the Nehari manifold requires that the corresponding nonlinearity is second order derivative about $u$ and $v$. Hence, in order to obtain the multiple $G$-symmetric solutions of system (1), the Nehari manifold techniques in the literature mentioned above are invalid and we need to look for other methods. To our knowledge, even in the particular case $\lambda=0$ and $p=2$, there are no results on the existence and multiplicity of $G$-symmetric solutions for system (1). It is therefore meaningful for us to investigate system (1) deeply. Let $\widetilde{Q}>0$ be a constant. Note that, here, we will try to treat both the cases of $\lambda=0, Q(x) \not \widetilde{Q}$ and $\lambda>0, Q(x) \equiv \widetilde{Q}$.

This paper is schemed as follows. In Section 2, we establish the appropriate Sobolev space which is applicable to the study of the elliptic system (1) and state the main results of this paper. In Section 3, we detail the proofs of several existence and multiplicity results for the case $\lambda=0$ and $Q(x) \not \widetilde{Q}$ in (1). In Section 4, we will present the proofs of multiplicity results for the case $\lambda>0$ and $Q(x) \not \widetilde{Q}$ in (1). Our methods in this paper are mainly based upon the symmetric criticality principle of Palais (see [26]) and variational arguments.

\section{Preliminaries and Main Results}

Let $O(\mathbb{N})$ be the group of orthogonal linear transformations in $\mathbb{R}^{N}$ and let $G \subset O(\mathbb{N})$ be a closed subgroup. For $x \neq 0$ we denote the cardinality of $G_{x}=\{g x ; g \in G\}$ by $\left|G_{x}\right|$ and set $|G|=\inf _{0 \neq x \in \mathbb{R}^{N}}\left|G_{x}\right|$. Note that, here, $|G|$ may be $+\infty$. We say that $f: \mathbb{R}^{N} \rightarrow \mathbb{R}$ is $G$-symmetric (or $G$-invariant) if $f(g x)=f(x)$ for every $g \in G$ and $x \in \mathbb{R}^{N}$ and in the context of Sobolev spaces this equality is understood a.e. on $\mathbb{R}^{N}$. In particular, if $f$ is radially symmetric, then the corresponding group $G$ is $O(\mathbb{N})$ and $|G|=+\infty$. We call $\Omega$ a $G$-symmetric subset of $\mathbb{R}^{N}$; if $x \in \Omega$, then $g x \in \Omega$ for all $g \in G$.

Let $\mathscr{D}^{1, p}\left(\mathbb{R}^{N}\right)$ denote the closure of $\mathscr{C}_{0}^{\infty}\left(\mathbb{R}^{N}\right)$ functions with respect to the norm $\left(\int_{\mathbb{R}^{N}}|\nabla u|^{p} d x\right)^{1 / p}$. We recall that the well-known Hardy inequality (see $[2,3]$ ) holds:

$$
\int_{\mathbb{R}^{N}} \frac{|u|^{p}}{|x|^{p}} d x \leq \frac{1}{\bar{\mu}} \int_{\mathbb{R}^{N}}|\nabla u|^{p} d x, \quad \forall u \in \mathscr{D}^{1, p}\left(\mathbb{R}^{N}\right),
$$

where $\bar{\mu}=((N-p) / p)^{p}$. For $\mu \in[0, \bar{\mu})$, we employ the following norm in $\mathscr{D}^{1, p}\left(\mathbb{R}^{N}\right)$ :

$$
\|u\|_{\mu}=\left[\int_{\mathbb{R}^{N}}\left(|\nabla u|^{p}-\mu \frac{|u|^{p}}{|x|^{p}}\right) d x\right]^{1 / p} .
$$

By inequality (5), we see that the above norm is equivalent to the usual norm $\left(\int_{\mathbb{R}^{N}}|\nabla u|^{p} d x\right)^{1 / p}$. The elliptic operator $\mathscr{L}_{p, \mu}=$ $-\operatorname{div}\left(|\nabla \cdot|^{p-2} \nabla \cdot\right)-\mu\left(\left.|\cdot|^{p-2} \cdot|| x\right|^{p}\right)$ is positive in $\mathscr{D}^{1, p}\left(\mathbb{R}^{N}\right)$ if $\mu \in[0, \bar{\mu})$. Moreover, we define the product space $\mathscr{D}^{1, p}\left(\mathbb{R}^{N}\right) \times$ $\mathscr{D}^{1, p}\left(\mathbb{R}^{N}\right)$ endowed with the norm

$$
\begin{array}{r}
\|(u, v)\|_{\mu}=\left(\|u\|_{\mu}^{p}+\|v\|_{\mu}^{p}\right)^{1 / p}, \\
\forall(u, v) \in \mathscr{D}^{1, p}\left(\mathbb{R}^{N}\right) \times \mathscr{D}^{1, p}\left(\mathbb{R}^{N}\right) .
\end{array}
$$

The natural functional space to study system (1) is the Banach space $\mathscr{D}_{G}^{1, p}\left(\mathbb{R}^{N}\right) \times \mathscr{D}_{G}^{1, p}\left(\mathbb{R}^{N}\right)$, which is the subspace of $\mathscr{D}^{1, p}\left(\mathbb{R}^{N}\right) \times \mathscr{D}^{1, p}\left(\mathbb{R}^{N}\right)$ consisting of all $G$-symmetric 
functions. Now in this paper, we are concerned with the following elliptic problems:

$$
\left(\begin{array}{rl}
\mathscr{P}_{\lambda}^{Q} \\
\lambda
\end{array}\right)\left\{\begin{array}{rlrl}
\mathscr{L}_{p, \mu} u=Q(x)\left(|u|^{p^{*}-2} u+\frac{\varsigma \alpha}{p^{*}}|u|^{\alpha-2} u|v|^{\beta}\right) & \\
& +\lambda h_{1}(x)|u|^{q-2} u, & & \text { in } \mathbb{R}^{N}, \\
\mathscr{L}_{p, \mu} v= & Q(x)\left(|v|^{p^{*}-2} v+\frac{\varsigma \beta}{p^{*}}|u|^{\alpha}|v|^{\beta-2} v\right) & \\
& +\lambda h_{2}(x)|v|^{q-2} v, & & \text { in } \mathbb{R}^{N}, \\
(u, v) \in \mathscr{D}_{G}^{1, p}\left(\mathbb{R}^{N}\right) \times \mathscr{D}_{G}^{1, p}\left(\mathbb{R}^{N}\right), & & u>0, \\
& & & v>0, \\
& & \text { in } \mathbb{R}^{N} .
\end{array}\right.
$$

To mention our main results, we need to introduce two notations $\mathscr{A}_{\mu}$ and $y_{\epsilon}(x)$, which are, respectively, defined by

$$
\begin{gathered}
\mathscr{A}_{\mu} \triangleq \inf _{u \in \mathscr{D}^{1, p}\left(\mathbb{R}^{N}\right) \backslash\{0\}} \frac{\int_{\mathbb{R}^{N}}\left(|\nabla u|^{p}-\mu\left(|u|^{p} /|x|^{p}\right)\right) d x}{\left(\int_{\mathbb{R}^{N}}|u|^{p^{*}} d x\right)^{p / p^{*}}} \\
y_{\epsilon}(x) \triangleq C \epsilon^{-9} U_{\mu}\left(\frac{|x|}{\epsilon}\right)
\end{gathered}
$$

where $\epsilon>0, \vartheta \triangleq(N-p) / p$, and the constant $C=$ $C(N, p, \mu)>0$, depending only on $N, p$, and $\mu$. From Kang [4], we see that $y_{\epsilon}(x)$ satisfies the equations

$$
\begin{gathered}
\left\|y_{\epsilon}\right\|_{\mu}^{p}=\int_{\mathbb{R}^{N}}\left(\left|\nabla y_{\epsilon}\right|^{p}-\mu \frac{\left|y_{\epsilon}\right|^{p}}{|x|^{p}}\right) d x=1, \\
\int_{\mathbb{R}^{N}} y_{\epsilon}^{p^{*}} d x=\int_{\mathbb{R}^{N}} y_{\epsilon}^{\alpha+\beta} d x=\mathscr{A}_{\mu}^{-(\alpha+\beta) / p} .
\end{gathered}
$$

The function $U_{\mu}>0$ in (10) is radially symmetric. Moreover, the following asymptotic properties at the origin and infinity for $U_{\mu}(r)$ and $U_{\mu}^{\prime}(r)$ hold [4]:

$$
\begin{array}{cl}
\lim _{r \rightarrow 0} r^{l_{1}} U_{\mu}(r)=C_{1}>0, & \lim _{r \rightarrow 0} r^{l_{1}+1}\left|U_{\mu}^{\prime}(r)\right|=C_{1} l_{1}>0, \\
\lim _{r \rightarrow+\infty} r^{l_{2}} U_{\mu}(r)=C_{2}>0, & \lim _{r \rightarrow+\infty} r^{l_{2}+1}\left|U_{\mu}^{\prime}(r)\right|=C_{2} l_{2}>0,
\end{array}
$$

where $C_{1}, C_{2}$ are positive constants and $l_{1}=l_{1}(N, p, \mu)$ and $l_{2}=l_{2}(N, p, \mu)$ are the zeroes of the function

$$
\mathscr{L}(t)=(p-1) t^{p}-(N-p) t^{p-1}+\mu, \quad t \geq 0, \quad 0 \leq \mu<\bar{\mu},
$$

satisfying

$$
0 \leq l_{1}<\vartheta<l_{2} \leq \frac{N-p}{p-1}, \quad \vartheta=\frac{N-p}{p} .
$$

We suppose that the functions $Q, h_{1}$, and $h_{2}$ verify the following hypotheses. (q.1) $Q \in \mathscr{C}\left(\mathbb{R}^{N}\right) \cap L^{\infty}\left(\mathbb{R}^{N}\right)$, and $Q$ is $G$-symmetric.

(q.2) $Q_{+} \not \equiv 0$, where $Q_{+}=\max \{0, Q\}$.

(h.1) $h_{1}$ and $h_{2}$ are $G$-symmetric.

(h.2) $h_{i}(x) \geq 0, h_{i}(x) \neq 0$, and $h_{i}(x) \in L^{\theta}\left(\mathbb{R}^{N}\right)$ with $\theta=$ $N p /(N p-q(N-p))$, where $i=1,2$.

The main results of this paper are summarized in the following.

Theorem 1. Suppose that (q.1) and (q.2) hold. If

$\int_{\mathbb{R}^{N}} Q(x) y_{\epsilon}^{\alpha+\beta} d x$

$$
\geq \max \left\{\frac{Q_{+}(0)}{\mathscr{A}_{\mu}^{(\alpha+\beta) / p}}, \frac{Q_{+}(\infty)}{\mathscr{A}_{\mu}^{(\alpha+\beta) / p}}, \frac{\left\|Q_{+}\right\|_{\infty}}{|G|^{(\alpha+\beta-p) / p} \mathscr{A}_{0}^{(\alpha+\beta) / p}}\right\}>0,
$$

for some $\epsilon>0$, where $Q_{+}(\infty)=\limsup _{|x| \rightarrow \infty} Q_{+}(x)$, then problem $\left(\mathscr{P}_{0}^{\mathrm{Q}}\right)$ has at least one positive solution in $\mathscr{D}_{G}^{1, p}\left(\mathbb{R}^{N}\right) \times$ $\mathscr{D}_{G}^{1, p}\left(\mathbb{R}^{N}\right)$.

Corollary 2. Suppose that (q.1) and (q.2) hold. Then we have the following statements.

(1) Problem $\left(\mathscr{P}_{0}^{\mathrm{Q}}\right)$ has a positive solution if

$$
Q(0)>0 \text {, }
$$

$Q(0) \geq \max \left\{Q_{+}(\infty),|G|^{(p-(\alpha+\beta)) / p}\left(\frac{\mathscr{A}_{0}}{\mathscr{A}_{\mu}}\right)^{-(\alpha+\beta) / p}\left\|Q_{+}\right\|_{\infty}\right\}$,

and either (i) $Q(x) \geq Q(0)+\Lambda_{0}|x|^{(\alpha+\beta)\left(l_{2}-9\right)}$ for some $\Lambda_{0}>0$ and $|x|$ small or (ii) $|Q(x)-Q(0)| \leq \Lambda_{1}|x|^{\kappa}$ for some constant $\Lambda_{1}>0, \kappa>(\alpha+\beta)\left(l_{2}-9\right)$ and $|x|$ small and

$$
\int_{\mathbb{R}^{N}}(Q(x)-Q(0))|x|^{-N-(\alpha+\beta)\left(l_{2}-9\right)} d x>0 .
$$

(2) Problem $\left(\mathscr{P}_{0}^{\mathrm{Q}}\right)$ admits at least one positive solution if $\lim _{|x| \rightarrow \infty} Q(x)=Q(\infty)$ exists and is positive,

$Q(\infty) \geq \max \left\{Q_{+}(0),|G|^{(p-(\alpha+\beta)) / p}\left(\frac{\mathscr{A}_{0}}{\mathscr{A}_{\mu}}\right)^{-(\alpha+\beta) / p}\left\|Q_{+}\right\|_{\infty}\right\}$,

and either (i) $Q(x) \geq Q(\infty)+\Lambda_{2}|x|^{-(\alpha+\beta)\left(\vartheta-l_{1}\right)}$ for some $\Lambda_{2}>0$ and large $|x|$ or (ii) $|Q(x)-Q(\infty)| \leq \Lambda_{3}|x|^{-\imath}$ for some constants $\Lambda_{3}>0, \iota>(\alpha+\beta)\left(\vartheta-l_{1}\right)$ and large $|x|$ and

$$
\int_{\mathbb{R}^{N}}(Q(x)-Q(\infty))|x|^{-N+(\alpha+\beta)\left(\vartheta-l_{1}\right)} d x>0 .
$$

(3) If $Q(x) \geq Q(\infty)=Q(0)>0$ on $\mathbb{R}^{N}$ and

$Q(\infty)=Q(0) \geq|G|^{(p-(\alpha+\beta)) / p}\left(\frac{\mathscr{A}_{0}}{\mathscr{A}_{\mu}}\right)^{-(\alpha+\beta) / p}\left\|Q_{+}\right\|_{\infty}$,

then problem $\left(\mathscr{P}_{0}^{\mathrm{Q}}\right)$ has at least one positive solution. 
Theorem 3. Suppose that $Q_{+}(0)=Q_{+}(\infty)=0$ and $|G|=$ $+\infty$. Then problem $\left(\mathscr{P}_{0}^{\mathrm{Q}}\right)$ has infinitely many G-symmetric solutions.

Corollary 4. If $Q$ is a radially symmetric function such that $Q_{+}(0)=Q_{+}(\infty)=0$, then problem $\left(\mathscr{P}_{0}^{\mathrm{Q}}\right)$ has infinitely many solutions which are radially symmetric.

Theorem 5. Let $\widetilde{Q}>0$ be a constant. Suppose that $Q(x) \equiv \widetilde{Q}$ and (h.1), (h.2) hold. Then there exists $\lambda^{*}>0$ such that, for any $\lambda \in\left(0, \lambda^{*}\right)$, problem $\left(\mathscr{P}_{\lambda}^{\widetilde{Q}}\right)$ possesses at least two positive solutions in $\mathscr{D}_{G}^{1, p}\left(\mathbb{R}^{N}\right) \times \mathscr{D}_{G}^{1, p}\left(\mathbb{R}^{N}\right)$.

Remark 6. The main results of this paper generalize, extend, and complement some results of the aforementioned papers $[9-12,24,25]$.

In the sequel, we denote by $\mathscr{D}_{G}^{1, p}\left(\mathbb{R}^{N}\right) \times \mathscr{D}_{G}^{1, p}\left(\mathbb{R}^{N}\right)$ the subspace of $\mathscr{D}^{1, p}\left(\mathbb{R}^{N}\right) \times \mathscr{D}^{1, p}\left(\mathbb{R}^{N}\right)$ consisting of all $G$ symmetric functions. The dual space of $\mathscr{D}_{G}^{1, p}\left(\mathbb{R}^{N}\right) \times \mathscr{D}_{G}^{1, p}\left(\mathbb{R}^{N}\right)$ $\left(\mathscr{D}^{1, p}\left(\mathbb{R}^{N}\right) \times \mathscr{D}^{1, p}\left(\mathbb{R}^{N}\right)\right.$, resp. $)$ is denoted by $\mathscr{D}_{G}^{-1, p^{\prime}}\left(\mathbb{R}^{N}\right) \times$ $\mathscr{D}_{G}^{-1, p^{\prime}}\left(\mathbb{R}^{N}\right)\left(\mathscr{D}^{-1, p^{\prime}}\left(\mathbb{R}^{N}\right) \times \mathscr{D}^{-1, p^{\prime}}\left(\mathbb{R}^{N}\right)\right.$, resp. $)$, where $1 / p+$ $1 / p^{\prime}=1$. The ball of center $x$ and radius $r$ is denoted by $B_{r}(x)$. We employ $C, C_{1}, C_{2}, \ldots$ to denote (possibly different) positive constants and denote by " $\rightarrow$ " convergence in norm in a given Banach space $X$ and by " $\rightarrow$ " weak convergence. Hereafter, $o_{n}(1)$ denotes a datum which tends to 0 as $n \rightarrow$ D. $L^{q}\left(\mathbb{R}^{N}, h(x)\right)$ denotes the weighted $L^{q}\left(\mathbb{R}^{N}\right)$ space with the norm $\left(\int_{\mathbb{R}^{N}} h(x)|u|^{q} d x\right)^{1 / q}$. A functional $J \in \mathscr{C}^{1}(X, \mathbb{R})$ is said to satisfy the $(P S)_{c}$ condition if each sequence $\left\{u_{n}\right\}$ in $X$ satisfying $J\left(u_{n}\right) \rightarrow c, J^{\prime}\left(u_{n}\right) \rightarrow 0$ in $X^{*}$ has a subsequence which strongly converges to some element in $X$.

\section{Existence and Multiplicity Results for Problem $\left(\mathscr{P}_{0}^{\mathrm{Q}}\right)$}

The corresponding energy functional of problem $\left(\mathscr{P}_{0}^{\mathrm{Q}}\right)$ is defined in $\mathscr{D}_{G}^{1, p}\left(\mathbb{R}^{N}\right) \times \mathscr{D}_{G}^{1, p}\left(\mathbb{R}^{N}\right)$ by

$$
\begin{aligned}
\mathscr{E}(u, v)= & \frac{1}{p}\|(u, v)\|_{\mu}^{p} \\
& -\frac{1}{p^{*}} \int_{\mathbb{R}^{N}} Q(x)\left(|u|^{p^{*}}+|v|^{p^{*}}+\varsigma|u|^{\alpha}|v|^{\beta}\right) d x .
\end{aligned}
$$

Note that (q.1) and (5) imply that $\mathscr{E} \in \mathscr{C}^{1}\left(\mathscr{D}_{G}^{1, p}\left(\mathbb{R}^{N}\right) \times\right.$ $\left.\mathscr{D}_{G}^{1, p}\left(\mathbb{R}^{N}\right), \mathbb{R}\right)$. It is well known that there exists a one-toone correspondence between the weak solutions of problem $\left(\mathscr{P}_{0}^{\mathrm{Q}}\right)$ and the critical points of $\mathscr{E}$. More precisely, any weak solution of $\left(\mathscr{P}_{0}^{\mathrm{Q}}\right)$ is exactly the critical point of $\mathscr{E}$ by the following symmetric principle (see Lemma 7); namely, $(u, v) \in \mathscr{D}_{G}^{1, p}\left(\mathbb{R}^{N}\right) \times \mathscr{D}_{G}^{1, p}\left(\mathbb{R}^{N}\right)$ satisfies $\left(\mathscr{P}_{0}^{\mathrm{Q}}\right)$ if and only if for all $\left(\varphi_{1}, \varphi_{2}\right) \in \mathscr{D}^{1, p}\left(\mathbb{R}^{N}\right) \times \mathscr{D}^{1, p}\left(\mathbb{R}^{N}\right)$, there holds

$$
\begin{gathered}
0=\int_{\mathbb{R}^{N}}\left(|\nabla u|^{p-2} \nabla u \nabla \varphi_{1}+|\nabla v|^{p-2} \nabla v \nabla \varphi_{2}\right. \\
\left.-\mu \frac{|u|^{p-2} u \varphi_{1}+|v|^{p-2} v \varphi_{2}}{|x|^{p}}\right) d x \\
-\int_{\mathbb{R}^{N}} Q(x)\left\{\left(|u|^{p^{*}-2} u+\frac{\varsigma \alpha}{p^{*}}|u|^{\alpha-2} u|v|^{\beta}\right) \varphi_{1}\right. \\
\left.+\left(|v|^{p^{*}-2} v+\frac{\varsigma \beta}{p^{*}}|u|^{\alpha}|v|^{\beta-2} v\right) \varphi_{2}\right\} d x .
\end{gathered}
$$

Lemma 7. $\mathscr{E}^{\prime}(u, v)=0$ in $\mathscr{D}_{G}^{-1, p^{\prime}}\left(\mathbb{R}^{N}\right) \times \mathscr{D}_{G}^{-1, p^{\prime}}\left(\mathbb{R}^{N}\right)$ implies $\mathscr{E}^{\prime}(u, v)=0$ in $\mathscr{D}^{-1, p^{\prime}}\left(\mathbb{R}^{N}\right) \times \mathscr{D}^{-1, p^{\prime}}\left(\mathbb{R}^{N}\right)$.

Proof. See the proof of [12, Lemma 1] (see also [25, Proposition 2.8]).

Now, for any $0 \leq \mu<\bar{\mu}, 0<\varsigma<+\infty, \alpha, \beta>1$, and $\alpha+\beta=p^{*}$, we define

$$
\begin{aligned}
& \mathscr{A}_{\mu, \varsigma}^{(\alpha, \beta)} \\
& \triangleq \inf _{u, v \in \mathscr{D}^{1, p}\left(\mathbb{R}^{N}\right) \backslash\{0\}} \frac{\int_{\mathbb{R}^{N}}\left(|\nabla u|^{p}+|\nabla v|^{p}-\mu\left(\left(|u|^{p}+|v|^{p}\right) /|x|^{p}\right)\right) d x}{\left[\int_{\mathbb{R}^{N}}\left(|u|^{p^{*}}+|v|^{p^{*}}+\varsigma|u|^{\alpha}|v|^{\beta}\right) d x\right]^{p /(\alpha+\beta)}}, \\
& K(\tau) \triangleq \frac{1+\tau^{p}}{\left(1+\varsigma \tau^{\beta}+\tau^{\alpha+\beta}\right)^{p /(\alpha+\beta)}}, \quad \tau \geq 0, \\
& K\left(\tau_{\min }\right) \triangleq \min _{\tau \geq 0} K(\tau)>0,
\end{aligned}
$$

where $\tau_{\min }>0$ is a minimal point of $K(\tau)$ and therefore a root of the equation

$$
(\alpha+\beta) \tau^{\alpha+\beta-p}+\varsigma \beta \tau^{\beta-p}-\varsigma \alpha \tau^{\beta}-(\alpha+\beta)=0, \quad \tau \geq 0 .
$$

Lemma 8. Suppose that $1<p<N, 0<\varsigma<+\infty$, and $0 \leq \mu<\bar{\mu}$. Then $\mathscr{A}_{\mu, \varsigma}^{(\alpha, \beta)}=K\left(\tau_{\min }\right) \mathscr{A}_{\mu}$, and $\mathscr{A}_{\mu, \varsigma}^{(\alpha, \beta)}$ has the minimizer $\left(y_{\epsilon}(x), \tau_{\min } y_{\epsilon}(x)\right), \forall \epsilon>0$, where $y_{\epsilon}(x)$ is the extremal function of $\mathscr{A}_{\mu}$ defined as in (10).

Proof. The proof is similar to the proof in Nyamoradi [16, Theorem 2].

Lemma 9. Let $\left\{\left(u_{n}, v_{n}\right)\right\}$ be a weakly convergent sequence to $(u, v)$ in $\mathscr{D}_{G}^{1, p}\left(\mathbb{R}^{N}\right) \times \mathscr{D}_{G}^{1, p}\left(\mathbb{R}^{N}\right)$ such that $\left|\nabla u_{n}\right|^{p} \rightarrow \eta^{(1)}$, $\left|\nabla v_{n}\right|^{p} \rightarrow \eta^{(2)},\left|u_{n}\right|^{p^{*}} \rightarrow \sigma^{(1)},\left|v_{n}\right|^{p^{*}} \rightarrow \sigma^{(2)},\left|u_{n}\right|^{\alpha}\left|v_{n}\right|^{\beta} \rightarrow v$, $|x|^{-p}\left|u_{n}\right|^{p} \rightarrow \gamma^{(1)}$, and $|x|^{-p}\left|v_{n}\right|^{p} \rightarrow \gamma^{(2)}$ in the sense of measures. Then there exists some at most countable set $\mathscr{J}$, $\left\{\eta_{j}^{(1)} \geq 0\right\}_{j \in \mathscr{F} \cup\{0\}},\left\{\eta_{j}^{(2)} \geq 0\right\}_{j \in \mathscr{J} \cup\{0\}},\left\{\sigma_{j}^{(1)} \geq 0\right\}_{j \in \mathscr{F} \cup\{0\}},\left\{\sigma_{j}^{(2)} \geq\right.$ $0\}_{j \in \mathcal{F} \cup\{0\}},\left\{v_{j} \geq 0\right\}_{j \in \mathcal{F} \cup\{0\}}, \gamma_{0}^{(1)} \geq 0, \gamma_{0}^{(2)} \geq 0$, and $\left\{x_{j}\right\}_{j \in \mathcal{F}} \subset$ $\mathbb{R}^{N} \backslash\{0\}$ such that 
(a) $\eta^{(1)} \geq|\nabla u|^{p}+\sum_{j \in \mathcal{g}} \eta_{j}^{(1)} \delta_{x_{j}}+\eta_{0}^{(1)} \delta_{0}, \eta^{(2)} \geq|\nabla v|^{p}+$ $\sum_{j \in \mathscr{J}} \eta_{j}^{(2)} \delta_{x_{j}}+\eta_{0}^{(2)} \delta_{0}$

(b) $\sigma^{(1)}=|u|^{p^{*}}+\sum_{j \in \mathcal{J}} \sigma_{j}^{(1)} \delta_{x_{j}}+\sigma_{0}^{(1)} \delta_{0}, \sigma^{(2)}=|v|^{p^{*}}+$ $\sum_{j \in \mathcal{J}} \sigma_{j}^{(2)} \delta_{x_{j}}+\sigma_{0}^{(2)} \delta_{0}$

(c) $v=|u|^{\alpha}|v|^{\beta}+\sum_{j \in \mathcal{F}} \nu_{j} \delta_{x_{j}}+v_{0} \delta_{0}, \gamma^{(1)}=\left(|u|^{p} /|x|^{p}\right)+$ $\gamma_{0}^{(1)} \delta_{0}, \gamma^{(2)}=\left(|v|^{p} /|x|^{p}\right)+\gamma_{0}^{(2)} \delta_{0}$,

(d) $\mathscr{A}_{0, \varsigma}^{(\alpha, \beta)}\left(\sigma_{j}^{(1)}+\sigma_{j}^{(2)}+\varsigma \nu_{j}\right)^{p /(\alpha+\beta)} \leq \eta_{j}^{(1)}+\eta_{j}^{(2)}$, $\mathscr{A}_{0}\left(\sigma_{j}^{(1)}\right)^{p / p^{*}} \leq \eta_{j}^{(1)}, \mathscr{A}_{0}\left(\sigma_{j}^{(2)}\right)^{p / p^{*}} \leq \eta_{j}^{(2)}$,

(e) $\mathscr{A}_{\mu, \varsigma}^{(\alpha, \beta)}\left(\sigma_{0}^{(1)}+\sigma_{0}^{(2)}+\varsigma \nu_{0}\right)^{p /(\alpha+\beta)} \leq \eta_{0}^{(1)}+\eta_{0}^{(2)}-\mu\left(\gamma_{0}^{(1)}+\gamma_{0}^{(2)}\right)$, $\mathscr{A}_{\mu}\left(\sigma_{0}^{(1)}\right)^{p / p^{*}} \leq \eta_{0}^{(1)}-\mu \gamma_{0}^{(1)}, \mathscr{A}_{\mu}\left(\sigma_{0}^{(2)}\right)^{p / p^{*}} \leq \eta_{0}^{(2)}-\mu \gamma_{0}^{(2)}$,

where $\delta_{x_{j}}, j \in \mathscr{J} \cup\{0\}$, is the Dirac-mass of 1 concentrated at $x_{j} \in \mathbb{R}^{N}$.

Proof. The proof is similar to that of the concentration compactness principle in $[27,28]$ (see also [20, Lemma 2.2]) and is omitted here.

In order to find critical points of $\mathscr{E}$, we need the following local $(P S)_{c}$ condition.

Lemma 10. Suppose that (q.1) and (q.2) hold. Then the (PS) condition in $\mathscr{D}_{G}^{1, p}\left(\mathbb{R}^{N}\right) \times \mathscr{D}_{G}^{1, p}\left(\mathbb{R}^{N}\right)$ holds for $\mathscr{E}$ if

$$
\begin{aligned}
c<c_{0}^{*} \triangleq & \frac{1}{N} \\
\times \min & \left\{\frac{\left(\mathscr{A}_{\mu, \varsigma}^{(\alpha, \beta)}\right)^{N / p}}{\left(Q_{+}(0)\right)^{(N-p) / p}}, \frac{\left(\mathscr{A}_{\mu, \varsigma}^{(\alpha, \beta)}\right)^{N / p}}{\left(Q_{+}(\infty)\right)^{(N-p) / p}},\right. \\
& \left.\frac{|G|\left(\mathscr{A}_{0, \varsigma}^{(\alpha, \beta)}\right)^{N / p}}{\left\|Q_{+}\right\|_{\infty}^{(N-p) / p}}\right\} .
\end{aligned}
$$

Proof. The proof is similar to that in [12, Proposition 2]. We sketch the argument here for completeness. Suppose $\left\{\left(u_{n}, v_{n}\right)\right\} \subset \mathscr{D}_{G}^{1, p}\left(\mathbb{R}^{N}\right) \times \mathscr{D}_{G}^{1, p}\left(\mathbb{R}^{N}\right)$ satisfies $\mathscr{E}\left(u_{n}, v_{n}\right) \rightarrow$ $c$ and $\mathscr{E}^{\prime}\left(u_{n}, v_{n}\right) \rightarrow 0$ with $c<c_{0}^{*}$. It is easy to show that $\left\{\left(u_{n}, v_{n}\right)\right\}$ is bounded in $\mathscr{D}_{G}^{1, p}\left(\mathbb{R}^{N}\right) \times \mathscr{D}_{G}^{1, p}\left(\mathbb{R}^{N}\right)$ and then $\left(u_{n}, v_{n}\right) \rightarrow(u, v)$ up to a subsequence. Moreover, we know from Lemma 9 that there exist measures $\eta^{(1)}, \eta^{(2)}, \sigma^{(1)}, \sigma^{(2)}$, $\nu, \gamma^{(1)}$, and $\gamma^{(2)}$ such that relations (a)-(e) of this lemma hold. Let $x_{j} \neq 0$ be a singular point of measures $\eta^{(1)}, \eta^{(2)}$, and $\nu$. As in [20], we can choose two functions $\phi_{1}, \phi_{2} \in \mathscr{C}^{1}\left(\mathbb{R}^{N}\right)$ such that $0 \leq \phi_{1}, \phi_{2} \leq 1, \phi_{1}=\phi_{2}=1$ for $\left|x-x_{j}\right| \leq \epsilon / 2, \phi_{1}=\phi_{2}=0$ for $\left|x-x_{j}\right| \geq \epsilon$ and $\left|\nabla \phi_{1}\right| \leq 4 / \epsilon,\left|\nabla \phi_{2}\right| \leq 4 / \epsilon$. By Lemma 7 , $\lim _{n \rightarrow \infty}\left\langle\mathscr{E}^{\prime}\left(u_{n}, v_{n}\right),\left(u_{n} \phi_{1}, v_{n} \phi_{2}\right)\right\rangle=0$, and, hence, using the Sobolev inequality and the Hölder inequality, we have

$$
\begin{aligned}
& \int_{\mathbb{R}^{N}}\left\{\left(\phi_{1} d \eta^{(1)}+\phi_{2} d \eta^{(2)}\right)-Q(x)\right. \\
& \left.\times\left(\phi_{1} d \sigma^{(1)}+\phi_{2} d \sigma^{(2)}\right)-\frac{\varsigma Q(x)}{p^{*}}\left(\alpha \phi_{1}+\beta \phi_{2}\right) d \nu\right\} \\
& -\int_{\mathbb{R}^{N}} \mu\left(\phi_{1} d \gamma^{(1)}+\phi_{2} d \gamma^{(2)}\right) \\
& \leq\left.\limsup _{n \rightarrow \infty} \int_{\mathbb{R}^{N}}\left|u_{n}\right| \nabla u_{n}\right|^{p-1} \nabla \phi_{1}+v_{n}\left|\nabla v_{n}\right|^{p-1} \nabla \phi_{2} \mid d x \\
& \leq \sup _{n \geq 1}\left(\int_{\mathbb{R}^{N}}\left|\nabla u_{n}\right|^{p} d x\right)^{(p-1) / p} \\
& \times \limsup _{n \rightarrow \infty}\left(\int_{\mathbb{R}^{N}}\left|u_{n}\right|^{p}\left|\nabla \phi_{1}\right|^{p} d x\right)^{1 / p} \\
& +\sup _{n \geq 1}\left(\int_{\mathbb{R}^{N}}\left|\nabla v_{n}\right|^{p} d x\right)^{(p-1) / p} \\
& \times \limsup _{n \rightarrow \infty}\left(\int_{\mathbb{R}^{N}}\left|v_{n}\right|^{p}\left|\nabla \phi_{2}\right|^{p} d x\right)^{1 / p} \\
& \leq C\left\{\left(\int_{\mathbb{R}^{N}}|u|^{p}\left|\nabla \phi_{1}\right|^{p} d x\right)^{1 / p}+\left(\int_{\mathbb{R}^{N}}|v|^{p}\left|\nabla \phi_{2}\right|^{p} d x\right)^{1 / p}\right\} \\
& \leq C\left\{\left(\int_{B_{\epsilon}\left(x_{j}\right)}|u|^{p^{*}} d x\right)^{1 / p^{*}}\left(\int_{\mathbb{R}^{N}}\left|\nabla \phi_{1}\right|^{N}\right)^{1 / N}\right. \\
& \left.+\left(\int_{B_{\epsilon}\left(x_{j}\right)}|v|^{p^{*}} d x\right)^{1 / p^{*}}\left(\int_{\mathbb{R}^{N}}\left|\nabla \phi_{2}\right|^{N}\right)^{1 / N}\right\} \\
& \leq C\left\{\left(\int_{B_{\epsilon}\left(x_{j}\right)}|\nabla u|^{p} d x\right)^{1 / p}+\left(\int_{B_{\epsilon}\left(x_{j}\right)}|\nabla v|^{p} d x\right)^{1 / p}\right\} .
\end{aligned}
$$

Taking limits as $\epsilon \rightarrow 0$ in (30), we obtain from Lemma 9 and the fact that $\alpha+\beta=p^{*}$ that

$$
Q\left(x_{j}\right)\left(\sigma_{j}^{(1)}+\sigma_{j}^{(2)}+\varsigma \nu_{j}\right) \geq \eta_{j}^{(1)}+\eta_{j}^{(2)} .
$$

The above inequality implies that the concentration of the measures $\sigma^{(1)}, \sigma^{(2)}$, and $v$ cannot occur at points where $Q\left(x_{j}\right) \leq 0$; that is, if $Q\left(x_{j}\right) \leq 0$ then $\sigma_{j}^{(1)}=\sigma_{j}^{(2)}=v_{j}=$ $\eta_{j}^{(1)}=\eta_{j}^{(2)}=0$. Combining (31) and (d) of Lemma 9 we infer that either (i) $\sigma_{j}^{(1)}=\sigma_{j}^{(2)}=v_{j}=0$ or (ii) $\sigma_{j}^{(1)}+\sigma_{j}^{(2)}+\varsigma v_{j} \geq$ $\left(\mathscr{A}_{0, \varsigma}^{(\alpha, \beta)} /\left\|Q_{+}\right\|_{\infty}\right)^{N / p}$. For the point $x=0$, similarly as in the case $x_{j} \neq 0$, we get

$$
\eta_{0}^{(1)}+\eta_{0}^{(2)}-\mu\left(\gamma_{0}^{(1)}+\gamma_{0}^{(2)}\right)-Q(0)\left(\sigma_{0}^{(1)}+\sigma_{0}^{(2)}+\varsigma \nu_{0}\right) \leq 0
$$

This, combined with (e) of Lemma 9, implies that either (iii) $\sigma_{0}^{(1)}=\sigma_{0}^{(2)}=\nu_{0}=0$ or (iv) $\sigma_{0}^{(1)}+\sigma_{0}^{(2)}+\varsigma \nu_{0} \geq\left(\mathscr{A}_{\mu, \varsigma}^{(\alpha, \beta)} / Q_{+}(0)\right)^{N / p}$. 
To study the concentration at infinity of the sequence we need to consider the following quantities:

(1) $\eta_{\infty}^{(1)}=\lim _{R \rightarrow \infty} \lim \sup _{n \rightarrow \infty} \int_{|x|>R}\left|\nabla u_{n}\right|^{p} d x, \eta_{\infty}^{(2)}=$ $\lim _{R \rightarrow \infty} \lim \sup _{n \rightarrow \infty} \int_{|x|>R}\left|\nabla v_{n}\right|^{p} d x$

(2) $\sigma_{\infty}^{(1)}=\lim _{R \rightarrow \infty} \lim \sup _{n \rightarrow \infty} \int_{|x|>R}\left|u_{n}\right|^{p^{*}} d x, \sigma_{\infty}^{(2)}=$ $\lim _{R \rightarrow \infty} \lim \sup _{n \rightarrow \infty} \int_{|x|>R}\left|v_{n}\right|^{p^{*}} d x$,

(3) $v_{\infty}=\lim _{R \rightarrow \infty} \lim \sup _{n \rightarrow \infty} \int_{|x|>R}\left|u_{n}\right|^{\alpha}\left|v_{n}\right|^{\beta} d x$,

(4) $\gamma_{\infty}^{(1)}=\lim _{R \rightarrow \infty} \lim \sup _{n \rightarrow \infty} \int_{|x|>R}\left(\left|u_{n}\right|^{p} /|x|^{p}\right) d x$, $\gamma_{\infty}^{(2)}=\lim _{R \rightarrow \infty} \lim \sup _{n \rightarrow \infty} \int_{|x|>R}\left(\left|v_{n}\right|^{p} /|x|^{p}\right) d x$.

Obviously, $\eta_{\infty}^{(1)}, \eta_{\infty}^{(2)}, v_{\infty}, \gamma_{\infty}^{(1)}$, and $\gamma_{\infty}^{(2)}$ exist and are finite. For $R>1$, let $\psi_{R}^{(1)}$ and $\psi_{R}^{(2)}$ be two regular functions such that $0 \leq \psi_{R}^{(1)}, \psi_{R}^{(2)} \leq 1, \psi_{R}^{(1)}=\psi_{R}^{(2)}=1$ for $|x|>R+1, \psi_{R}^{(1)}=$ $\psi_{R}^{(2)}=0$ for $|x|<R$ and $\left|\nabla \psi_{R}^{(1)}\right| \leq 4 / R,\left|\nabla \psi_{R}^{(2)}\right| \leq 4 / R$. Since the sequence $\left\{\left(u_{n} \psi_{R}^{(1)}, v_{n} \psi_{R}^{(2)}\right)\right\}$ is bounded in $\mathscr{D}^{1, p}\left(\mathbb{R}^{N}\right) \times$ $\mathscr{D}^{1, p}\left(\mathbb{R}^{N}\right)$, we get from (23) that

$$
\begin{aligned}
& 0=\lim _{n \rightarrow \infty}\left\langle\mathscr{E}^{\prime}\left(u_{n}, v_{n}\right),\left(u_{n} \psi_{R}^{(1)}, v_{n} \psi_{R}^{(2)}\right)\right\rangle \\
&=\lim _{n \rightarrow \infty}\left\{\int _ { \mathbb { R } ^ { N } } \left(\left|\nabla u_{n}\right|^{p} \psi_{R}^{(1)}+\left|\nabla v_{n}\right|^{p} \psi_{R}^{(2)}\right.\right. \\
&\left.-\mu \frac{\left|u_{n}\right|^{p} \psi_{R}^{(1)}+\left|v_{n}\right|^{p} \psi_{R}^{(2)}}{|x|^{p}}\right) d x \\
&+\int_{\mathbb{R}^{N}}\left(u_{n}\left|\nabla u_{n}\right|^{p-2} \nabla u_{n} \nabla \psi_{R}^{(1)}\right. \\
&\left.+v_{n}\left|\nabla v_{n}\right|^{p-2} \nabla v_{n} \nabla \psi_{R}^{(2)}\right) d x \\
& \quad \int_{\mathbb{R}^{N}}(x)\left[\left|u_{n}\right|^{p^{*}} \psi_{R}^{(1)}+\left|v_{n}\right|^{p^{*}} \psi_{R}^{(2)}\right. \\
&+\frac{\varsigma}{p^{*}}\left|u_{n}\right|^{\alpha}\left|v_{n}\right|^{\beta} \\
&\left.\left.\times\left(\alpha \psi_{R}^{(1)}+\beta \psi_{R}^{(2)}\right)\right] d x\right\} .
\end{aligned}
$$

We now observe that $u_{n} \rightarrow u$ in $L^{p}(R<|x|<R+1)$. Therefore, using the Sobolev inequality and the Hölder inequality we can easily check that

$$
\begin{aligned}
& \left.\lim _{R \rightarrow \infty} \limsup _{n \rightarrow \infty}\left|\int_{\mathbb{R}^{N}} u_{n}\right| \nabla u_{n}\right|^{p-2} \nabla u_{n} \nabla \psi_{R}^{(1)} d x \mid=0, \\
& \left.\lim _{R \rightarrow \infty} \limsup _{n \rightarrow \infty}\left|\int_{\mathbb{R}^{N}} v_{n}\right| \nabla v_{n}\right|^{p-2} \nabla v_{n} \nabla \psi_{R}^{(2)} d x \mid=0 .
\end{aligned}
$$

Consequently, taking into account the definitions (1)-(4) of $\eta_{\infty}^{(1)}, \eta_{\infty}^{(2)}, \sigma_{\infty}^{(1)}, \sigma_{\infty}^{(2)}, v_{\infty}, \gamma_{\infty}^{(1)}$, and $\gamma_{\infty}^{(2)}$, we deduce from (33) and (34) that

$$
Q(\infty)\left(\sigma_{\infty}^{(1)}+\sigma_{\infty}^{(2)}+\varsigma \nu_{\infty}\right) \geq \eta_{\infty}^{(1)}+\eta_{\infty}^{(2)}-\mu\left(\gamma_{\infty}^{(1)}+\gamma_{\infty}^{(2)}\right) .
$$

On the other hand, by (5) and the definition (25) of $\mathscr{A}_{\mu, \varsigma}^{(\alpha, \beta)}$ we easily see that $\bar{\mu} \gamma_{\infty}^{(1)} \leq \eta_{\infty}^{(1)}, \bar{\mu} \gamma_{\infty}^{(2)} \leq \eta_{\infty}^{(2)}$ and

$$
\mathscr{A}_{\mu, \varsigma}^{(\alpha, \beta)}\left(\sigma_{\infty}^{(1)}+\sigma_{\infty}^{(2)}+\varsigma \nu_{\infty}\right)^{p /(\alpha+\beta)} \leq \eta_{\infty}^{(1)}+\eta_{\infty}^{(2)}-\mu\left(\gamma_{\infty}^{(1)}+\gamma_{\infty}^{(2)}\right) .
$$

This, combined with (35), implies that either (v) $\sigma_{\infty}^{(1)}=\sigma_{\infty}^{(2)}=$ $\nu_{\infty}=0$ or (vi) $\sigma_{\infty}^{(1)}+\sigma_{\infty}^{(2)}+\varsigma \nu_{\infty} \geq\left(\mathscr{A}_{\mu, \varsigma}^{(\alpha, \beta)} / Q_{+}(\infty)\right)^{N / p}$. We now rule out the cases (ii), (iv), and (vi). For every continuous nonnegative function $\psi$ such that $0 \leq \psi(x) \leq 1$ on $\mathbb{R}^{N}$, we obtain from (23) and (24) that

$$
\begin{gathered}
c=\lim _{n \rightarrow \infty}\left(\mathscr{E}\left(u_{n}, v_{n}\right)-\frac{1}{p^{*}}\left\langle\mathscr{E}^{\prime}\left(u_{n}, v_{n}\right),\left(u_{n}, v_{n}\right)\right\rangle\right) \\
=\frac{1}{N} \lim _{n \rightarrow \infty} \int_{\mathbb{R}^{N}}\left(\left|\nabla u_{n}\right|^{p}+\left|\nabla v_{n}\right|^{p}-\mu \frac{\left|u_{n}\right|^{p}+\left|v_{n}\right|^{p}}{|x|^{p}}\right) d x \\
\geq \frac{1}{N} \limsup _{n \rightarrow \infty} \int_{\mathbb{R}^{N}}\left(\left|\nabla u_{n}\right|^{p}+\left|\nabla v_{n}\right|^{p}\right. \\
\left.-\mu \frac{\left|u_{n}\right|^{p}+\left|v_{n}\right|^{p}}{|x|^{p}}\right) \psi(x) d x .
\end{gathered}
$$

If (ii) occurs, then the set $\mathscr{J}$ must be finite because the measures $\sigma^{(1)}, \sigma^{(2)}$, and $v$ are bounded. Since functions $\left(u_{n}, v_{n}\right)$ are $G$-symmetric, the measures $\sigma^{(1)}, \sigma^{(2)}$, and $v$ must be $G$-invariant. This means that if $x_{j} \neq 0$ is a singular point of $\sigma^{(1)}, \sigma^{(2)}$, and $\nu$, so is $g x_{j}$ for each $g \in G$, and the mass of $\sigma^{(1)}, \sigma^{(2)}$, and $v$ concentrated at $g x_{j}$ is the same for each $g \in G$. If we assume the existence of $j \in \mathscr{J}$ with $x_{j} \neq 0$ such that (ii) holds, then we choose $\psi$ with compact support so that $\psi\left(g x_{j}\right)=1$ for each $g \in G$ and we obtain

$$
\begin{aligned}
c & \geq \frac{1}{N}|G|\left(\eta_{j}^{(1)}+\eta_{j}^{(2)}\right) \\
& \geq \frac{1}{N}|G| \mathscr{A}_{0, \varsigma}^{(\alpha, \beta)}\left(\sigma_{j}^{(1)}+\sigma_{j}^{(2)}+\varsigma \nu_{j}\right)^{p /(\alpha+\beta)} \\
& \geq \frac{1}{N}|G|\left(\mathscr{A}_{0, \varsigma}^{(\alpha, \beta)}\right)^{N / p}\left\|Q_{+}\right\|_{\infty}^{-(N-p) / p},
\end{aligned}
$$

which contradicts (29). Similarly, if (iv) holds for $x=0$, we choose $\psi$ with compact support, so that $\psi(0)=1$ and we have

$$
\begin{aligned}
c & \geq \frac{1}{N}\left(\eta_{0}^{(1)}+\eta_{0}^{(2)}-\mu \gamma_{0}^{(1)}-\mu \gamma_{0}^{(2)}\right) \\
& \geq \frac{1}{N} \mathscr{A}_{\mu, \varsigma}^{(\alpha, \beta)}\left(\sigma_{0}^{(1)}+\sigma_{0}^{(2)}+\varsigma \nu_{0}\right)^{p /(\alpha+\beta)} \\
& \geq \frac{1}{N}\left(\mathscr{A}_{\mu, \varsigma}^{(\alpha, \beta)}\right)^{N / p}\left(Q_{+}(0)\right)^{-(N-p) / p},
\end{aligned}
$$


which is impossible. Finally, if (vi) occurs at $\infty$, we take $\psi=$ $\psi_{R}^{(1)}=\psi_{R}^{(2)}$ to get

$$
\begin{aligned}
c & \geq \frac{1}{N}\left(\eta_{\infty}^{(1)}+\eta_{\infty}^{(2)}-\mu \gamma_{\infty}^{(1)}-\mu \gamma_{\infty}^{(2)}\right) \\
& \geq \frac{1}{N} \mathscr{A}_{\mu, \varsigma}^{(\alpha, \beta)}\left(\sigma_{\infty}^{(1)}+\sigma_{\infty}^{(2)}+\varsigma \nu_{\infty}\right)^{p /(\alpha+\beta)} \\
& \geq \frac{1}{N}\left(\mathscr{A}_{\mu, \varsigma}^{(\alpha, \beta)}\right)^{N / p}\left(Q_{+}(\infty)\right)^{-(N-p) / p}
\end{aligned}
$$

a contradiction with (29). Consequently, $\sigma_{j}^{(1)}=\sigma_{j}^{(2)}=v_{j}=0$ for all $j \in \mathscr{J} \cup\{0, \infty\}$ and this implies that

$$
\begin{gathered}
\lim _{n \rightarrow \infty} \int_{\mathbb{R}^{N}}\left(\left|u_{n}\right|^{p^{*}}+\left|v_{n}\right|^{p^{*}}+\varsigma\left|u_{n}\right|^{\alpha}\left|v_{n}\right|^{\beta}\right) d x \\
=\int_{\mathbb{R}^{N}}\left(|u|^{p^{*}}+|v|^{p^{*}}+\varsigma|u|^{\alpha}|v|^{\beta}\right) d x .
\end{gathered}
$$

Finally, observe that $\mathscr{E}^{\prime}(u, v)=0$ and, hence, by $\lim _{n \rightarrow \infty}\left\langle\mathscr{E}^{\prime}\left(u_{n}, v_{n}\right)-\mathscr{E}^{\prime}(u, v),\left(u_{n}-u, v_{n}-v\right)\right\rangle=0$, we obtain $\left(u_{n}, v_{n}\right) \rightarrow(u, v)$ as $n \rightarrow \infty$ in $\mathscr{D}^{1, p}\left(\mathbb{R}^{N}\right) \times \mathscr{D}^{1, p}\left(\mathbb{R}^{N}\right)$. The assertion follows.

As an immediate consequence of Lemma 10 we obtain the following result.

Corollary 11. If $Q_{+}(0)=Q_{+}(\infty)=0$ and $|G|=+\infty$, then the functional $\mathscr{E}$ satisfies the $(P S)_{c}$ condition for every $c \in \mathbb{R}$.

Proof of Theorem 1. Firstly, we choose $\epsilon>0$ such that the condition (17) holds, where $y_{\epsilon}$ is the extremal function satisfying (10), (11), and (12). By (q.1), (23), and (25), we have

$$
\begin{aligned}
\mathscr{E}(u, v)= & \frac{1}{p}\|(u, v)\|_{\mu}^{p} \\
& -\frac{1}{p^{*}} \int_{\mathbb{R}^{N}} Q(x)\left(|u|^{p^{*}}+|v|^{p^{*}}+\varsigma|u|^{\alpha}|v|^{\beta}\right) d x \\
\geq & \frac{1}{p}\|(u, v)\|_{\mu}^{p}-\frac{1}{p^{*}}\|Q\|_{\infty}\left(\mathscr{A}_{\mu, \varsigma}^{(\alpha, \beta)}\right)^{-(\alpha+\beta) / p}\|(u, v)\|_{\mu}^{\alpha+\beta} .
\end{aligned}
$$

Hence there exist constants $\alpha_{0}>0$ and $\rho>0$ such that $\mathscr{E}(u, v)$ $\geq \alpha_{0}$ for all $\|(u, v)\|_{\mu}=\rho$. Furthermore, if we set $u=y_{\epsilon}$, $v=\tau_{\min } y_{\epsilon}$ and

$$
\begin{aligned}
\Phi(t)= & \mathscr{E}(t u, t v)=\mathscr{E}\left(t y_{\epsilon}, t \tau_{\min } y_{\epsilon}\right) \\
= & \frac{t^{p}}{p} \int_{\mathbb{R}^{N}}\left(1+\tau_{\min }^{p}\right)\left(\left|\nabla y_{\epsilon}\right|^{p}-\mu \frac{\left|y_{\epsilon}\right|^{p}}{|x|^{p}}\right) d x \\
& -\frac{t^{p^{*}}}{p^{*}} \int_{\mathbb{R}^{N}}\left(1+\varsigma \tau_{\min }^{\beta}+\tau_{\min }^{\alpha+\beta}\right) Q(x) y_{\epsilon}^{\alpha+\beta} d x,
\end{aligned}
$$

with $t \geq 0$, then we can check that $\Phi(t)$ has a unique maximum at some $\bar{t}>0$. A simple computation gives us the value

$$
\bar{t}=\left\{\frac{\left(1+\tau_{\min }^{p}\right) \int_{\mathbb{R}^{N}}\left(\left|\nabla y_{\epsilon}\right|^{p}-\mu\left(\left|y_{\epsilon}\right|^{p} /|x|^{p}\right)\right) d x}{\left(1+\varsigma \tau_{\min }^{\beta}+\tau_{\min }^{\alpha+\beta}\right) \int_{\mathbb{R}^{N}} Q(x) y_{\epsilon}^{\alpha+\beta} d x}\right\}^{1 /(\alpha+\beta-p)} .
$$

Consequently, we obtain from (26) and (27) that

$\max _{t \geq 0} \Phi(t)$

$$
\begin{aligned}
& =\mathscr{E}\left(\bar{t} y_{\epsilon}, \bar{t} \tau_{\min } y_{\epsilon}\right)=\frac{1}{N} \\
& \times\left\{\frac{K\left(\tau_{\min }\right) \int_{\mathbb{R}^{N}}\left(\left|\nabla y_{\epsilon}\right|^{p}-\mu\left(\left|y_{\epsilon}\right|^{p} /|x|^{p}\right)\right) d x}{\left(\int_{\mathbb{R}^{N}} Q(x) y_{\epsilon}^{\alpha+\beta} d x\right)^{p /(\alpha+\beta)}}\right\}
\end{aligned}
$$

Since $\Phi(t) \rightarrow-\infty$ as $t \rightarrow \infty$, we can choose $t_{0}>0$ such that $\mathscr{E}\left(t_{0} y_{\epsilon}, t_{0} \tau_{\min } y_{\epsilon}\right)<0$ and $\left\|\left(t_{0} y_{\epsilon}, t_{0} \tau_{\min } y_{\epsilon}\right)\right\|_{\mu}>\rho$ and set

$$
c_{0}=\inf _{\gamma \in \Gamma \in[0,1]} \max _{t \in[} \mathscr{E}(\gamma(t)),
$$

where

$$
\begin{gathered}
\Gamma=\left\{\gamma \in \mathscr{C}\left([0,1], \mathscr{D}_{G}^{1, p}\left(\mathbb{R}^{N}\right) \times \mathscr{D}_{G}^{1, p}\left(\mathbb{R}^{N}\right)\right) ;\right. \\
\left.\gamma(0)=(0,0), \gamma(1)=\left(t_{0} y_{\epsilon}, t_{0} \tau_{\min } y_{\epsilon}\right)\right\} .
\end{gathered}
$$

From (11), (17), (29), (45), (46), and Lemma 8, we obtain that

$$
\begin{aligned}
c_{0} & \leq \mathscr{E}\left(\bar{t} y_{\epsilon}, \bar{t} \tau_{\min } y_{\epsilon}\right) \\
& \left.=\frac{1}{N}\left\{\frac{K\left(\tau_{\min }\right) \int_{\mathbb{R}^{N}}\left(\left|\nabla y_{\epsilon}\right|^{p}-\mu\left(\left|y_{\epsilon}\right|^{p} /|x|^{p}\right)\right) d x}{\left(\int_{\mathbb{R}^{N}} Q(x) y_{\epsilon}^{\alpha+\beta} d x\right)^{p /(\alpha+\beta)}}\right\}\right\}^{(\alpha+\beta) /(\alpha+\beta-p)} \\
& \leq \frac{1}{N}\left\{\frac{K\left(\tau_{\min }\right) \int_{\mathbb{R}^{N}}\left(\left|\nabla y_{\epsilon}\right|^{p}-\mu\left(\left|y_{\epsilon}\right|^{p} /|x|^{p}\right)\right) d x}{\left(\max \left\{Q_{+}(0) / \mathscr{A}_{\mu}^{(\alpha+\beta) / p}, Q_{+}(\infty) / \mathscr{A}_{\mu}^{(\alpha+\beta) / p},\left\|Q_{+}\right\|_{\infty} /|G|^{(\alpha+\beta-p) / p} \mathscr{A}_{0}^{(\alpha+\beta) / p}\right\}\right)^{p /(\alpha+\beta)}}\right\}^{(\alpha+\beta) /(\alpha+\beta-p)} \\
& =\frac{1}{N} \min \left\{\frac{\left(\mathscr{A}_{\mu, \varsigma}^{(\alpha, \beta)}\right)^{N / p}}{\left(Q_{+}(0)\right)^{(N-p) / p}}, \frac{\left(\mathscr{A}_{\mu, \varsigma}^{(\alpha, \beta)}\right)^{N / p}}{\left(Q_{+}(\infty)\right)^{(N-p) / p}}, \frac{|G|\left(\mathscr{A}_{0, \varsigma}^{(\alpha, \beta)}\right)^{N / p}}{\left\|Q_{+}\right\|_{\infty}^{(N-p) / p}}\right\}=c_{0}^{*} .
\end{aligned}
$$


If $c_{0}<c_{0}^{*}$, then we conclude from Lemma 10 that the $(P S)_{c}$ condition holds and the conclusion follows by the mountain pass theorem in [29] (see also [30]). If $c_{0}=c_{0}^{*}$, then $\gamma(t)=$ $\left(t t_{0} y_{\epsilon}, t t_{0} \tau_{\min } y_{\epsilon}\right)$, with $0 \leq t \leq 1$, is a path in $\Gamma$ such that $\max _{t \in[0,1]} \mathscr{E}(\gamma(t))=c_{0}$. Thus, either $\Phi^{\prime}(\bar{t})=\mathscr{E}^{\prime}\left(\bar{t} y_{\epsilon}, \bar{t} \tau_{\min } y_{\epsilon}\right)$ $=0$ and we are done, or $\gamma$ can be deformed to a path $\tilde{\gamma} \in \Gamma$ with $\max _{t \in[0,1]} \mathscr{E}(\widetilde{\gamma}(t))<c_{0}$, which is impossible. Hence we have a nontrivial solution $\left(u_{0}, v_{0}\right) \in \mathscr{D}_{G}^{1, p}\left(\mathbb{R}^{N}\right) \times \mathscr{D}_{G}^{1, p}\left(\mathbb{R}^{N}\right)$ to problem $\left(\mathscr{P}_{0}^{\mathrm{Q}}\right)$. In the following, we have just to show that the solution $\left(u_{0}, v_{0}\right)$ can be chosen to be positive on $\mathbb{R}^{N}$. Consider the Nehari manifold

$$
\begin{gathered}
\mathscr{M}_{\mu} \triangleq\left\{(u, v) \in \mathscr{D}_{G}^{1, p}\left(\mathbb{R}^{N}\right) \times \mathscr{D}_{G}^{1, p}\left(\mathbb{R}^{N}\right) \backslash\{(0,0)\}\right. \\
\left.\left\langle\mathscr{E}^{\prime}(u, v),(u, v)\right\rangle=0\right\} .
\end{gathered}
$$

Writing an arbitrary element $(u, v) \in \mathscr{M}_{\mu}$ as $(u, v)=$ $t(\widetilde{u}, \widetilde{v})(t>0)$, with $\|(\widetilde{u}, \widetilde{v})) \|_{\mu}=1$, we deduce from (24), (25), and the fact that $\left\langle\mathscr{E}^{\prime}(t \tilde{u}, t \widetilde{v}),(t \tilde{u}, t \widetilde{v})\right\rangle=0$ that

$$
\begin{aligned}
0 & =1-t^{p^{*}-p} \int_{\mathbb{R}^{N}} Q(x)\left(|\widetilde{u}|^{p^{*}}+|\widetilde{v}|^{p^{*}}+\varsigma|\widetilde{u}|^{\alpha}|\widetilde{v}|^{\beta}\right) d x \\
& \geq 1-\left(\mathscr{A}_{\mu, \varsigma}^{(\alpha, \beta)}\right)^{-(\alpha+\beta) / p}\|Q\|_{\infty}\|(\widetilde{u}, \widetilde{v})\|_{\mu}^{(\alpha+\beta) / p} t^{p^{*}-p} \\
& \geq 1-C t^{p^{*}-p} .
\end{aligned}
$$

This implies that $t \geq \xi_{0}$, with a constant $\xi_{0}>0$ independent of $(u, v)$. Thus we conclude that the set $\mathscr{M}_{\mu}$ is bounded away from 0 and $\inf _{(u, v) \in M_{\mu}} \mathscr{E}(u, v)>0$. Set

$$
\begin{aligned}
\mathscr{G}(u, v) & =\left\langle\mathscr{E}^{\prime}(u, v),(u, v)\right\rangle \\
& =\|(u, v)\|_{\mu}^{p}-\int_{\mathbb{R}^{N}} Q(x)\left(|u|^{p^{*}}+|v|^{p^{*}}+\varsigma|u|^{\alpha}|v|^{\beta}\right) d x .
\end{aligned}
$$

Then $\left\langle\mathscr{G}^{\prime}(u, v),(u, v)\right\rangle=\left(p-p^{*}\right)\|(u, v)\|_{\mu}^{p} \neq 0$, and, hence, $\mathscr{M}_{\mu}$ is a $\mathscr{C}^{1}$-manifold. Notice that $\left(u_{0}, v_{0}\right) \in \mathscr{M}_{\mu}$ and set $\bar{c}_{0}=$ $\inf _{(u, v) \in \mathscr{M}_{\mu}} \mathscr{E}(u, v)$. We now claim $\bar{c}_{0}=c_{0}$. Indeed, if $\bar{c}_{0}<c_{0}$, then we can find $\left(u_{0}^{*}, v_{0}^{*}\right) \in \mathscr{M}_{\mu}$ such that $\mathscr{E}\left(u_{0}^{*}, v_{0}^{*}\right)<c_{0}$. Consequently, we deduce that

$$
\int_{\mathbb{R}^{N}} Q(x)\left(\left|u_{0}^{*}\right|^{p^{*}}+\left|v_{0}^{*}\right|^{p^{*}}+\varsigma\left|u_{0}^{*}\right|^{\alpha}\left|v_{0}^{*}\right|^{\beta}\right) d x=\left\|\left(u_{0}^{*}, v_{0}^{*}\right)\right\|_{\mu}^{p}>0 .
$$

By a straightforward calculation, we get

$$
\begin{array}{rl}
\sup _{t \geq 0} & \mathscr{E}\left(t u_{0}^{*}, t v_{0}^{*}\right) \\
& =\mathscr{E}\left(u_{0}^{*}, v_{0}^{*}\right) \\
& =\frac{1}{N} \int_{\mathbb{R}^{N}} Q(x)\left(\left|u_{0}^{*}\right|^{p^{*}}+\left|v_{0}^{*}\right|^{p^{*}}+\varsigma\left|u_{0}^{*}\right|^{\alpha}\left|v_{0}^{*}\right|^{\beta}\right) d x<c_{0} .
\end{array}
$$

Setting $\gamma(t)=\left(t t_{0}^{*} u_{0}^{*}, t t_{0}^{*} v_{0}^{*}\right)$ for $t \in[0,1]$, with $t_{0}^{*}$ such that $\left\|\left(t_{0}^{*} u_{0}^{*}, t_{0}^{*} v_{0}^{*}\right)\right\|_{\mu}>\rho$, we obtain that
$\mathscr{E}(\gamma(t))=\mathscr{E}\left(t t_{0}^{*} u_{0}^{*}, t t_{0}^{*} v_{0}^{*}\right)<c_{0}$ on $[0,1]$, a contradiction with the definition (46) of $c_{0}$. Hence we have $\bar{c}_{0}=c_{0}$. Finally, by the strong maximum principle, we obtain $u_{0}>0$ and $v_{0}>0$ on $\mathbb{R}^{N}$. This, combined with (24) and Lemma 7, implies that $\left(u_{0}, v_{0}\right)$ is a positive $G$-symmetric solution of $\left(\mathscr{P}_{0}^{\mathrm{Q}}\right)$.

Proof of Corollary 2. First of all, we observe that, due to the identity (12), inequality (17) is equivalent to $\int_{\mathbb{R}^{N}}(Q(x)-$ Q) $y_{\epsilon}^{\alpha+\beta} d x \geq 0$ for some $\epsilon>0$, or equivalently

$$
\epsilon^{-(\alpha+\beta) \vartheta} \int_{\mathbb{R}^{N}}(Q(x)-\bar{Q})\left[U_{\mu}\left(\frac{|x|}{\epsilon}\right)\right]^{\alpha+\beta} d x \geq 0
$$

for some $\epsilon>0$, where $\bar{Q}=\max \left\{Q_{+}(0), Q_{+}(\infty)\right.$, $\left.|G|^{(p-(\alpha+\beta)) / p}\left(\mathscr{A}_{0} / \mathscr{A}_{\mu}\right)^{-(\alpha+\beta) / p}\left\|Q_{+}\right\|_{\infty}\right\}$ and $\vartheta=(N-p) / p$. Part (1), case (i): according to (54), we need to show that

$$
\epsilon^{-(\alpha+\beta) l_{2}} \int_{\mathbb{R}^{N}}(Q(x)-Q(0))\left[U_{\mu}\left(\frac{|x|}{\epsilon}\right)\right]^{\alpha+\beta} d x \geq 0
$$

for some $\epsilon>0$. We choose $\varrho_{0}>0$ so that $Q(x) \geq Q(0)+$ $\Lambda_{0}|x|^{(\alpha+\beta)\left(l_{2}-9\right)}$ for $|x| \leq \varrho_{0}$. This, combined with (14) and (16) and the fact that $-(\alpha+\beta) \vartheta=-N$, implies that

$$
\begin{aligned}
& \epsilon^{-(\alpha+\beta) l_{2}} \int_{|x| \leq \varrho_{0}}(Q(x)-Q(0))\left[U_{\mu}\left(\frac{|x|}{\epsilon}\right)\right]^{\alpha+\beta} d x \\
& \geq \Lambda_{0} \int_{|x| \leq \varrho_{0}}|x|^{-(\alpha+\beta) \vartheta}\left[\left(\frac{|x|}{\epsilon}\right)^{l_{2}} U_{\mu}\left(\frac{|x|}{\epsilon}\right)\right]^{\alpha+\beta} d x \rightarrow+\infty,
\end{aligned}
$$

as $\epsilon \rightarrow 0$. On the other hand, for all $\epsilon>0$, we have

$$
\begin{aligned}
& \epsilon^{-(\alpha+\beta) l_{2}} \int_{|x|>\varrho_{0}}|Q(x)-Q(0)|\left[U_{\mu}\left(\frac{|x|}{\epsilon}\right)\right]^{\alpha+\beta} d x \\
& \leq \int_{|x|>\varrho_{0}} \frac{|Q(x)-Q(0)|}{|x|^{(\alpha+\beta) l_{2}}}\left[\left(\frac{|x|}{\epsilon}\right)^{l_{2}} U_{\mu}\left(\frac{|x|}{\epsilon}\right)\right]^{\alpha+\beta} d x \leq \widetilde{C_{1}},
\end{aligned}
$$

for some constant $\widetilde{C_{1}}>0$ independent of $\epsilon$. Combining (56) and (57), we get (55) for $\epsilon$ sufficiently small.

Part (1), case (ii): we choose $\varrho_{1}>0$ so that $|Q(x)-Q(0)| \leq$ $\Lambda_{1}|x|^{\kappa}$ for $|x| \leq \varrho_{1}$. Since $\kappa>(\alpha+\beta)\left(l_{2}-9\right)>0$, we deduce from (14) and the fact that $(\alpha+\beta) l_{2}=N+(\alpha+\beta)\left(l_{2}-\vartheta\right)$ that

$$
\begin{aligned}
& \epsilon^{-(\alpha+\beta) l_{2}} \int_{\mathbb{R}^{N}}|Q(x)-Q(0)|\left[U_{\mu}\left(\frac{|x|}{\epsilon}\right)\right]^{\alpha+\beta} d x \\
& =\int_{\mathbb{R}^{N}} \frac{|Q(x)-Q(0)|}{|x|^{(\alpha+\beta) l_{2}}}\left[\left(\frac{|x|}{\epsilon}\right)^{l_{2}} U_{\mu}\left(\frac{|x|}{\epsilon}\right)\right]^{\alpha+\beta} d x \\
& \leq C \int_{\mathbb{R}^{N}} \frac{|Q(x)-Q(0)|}{|x|^{N+(\alpha+\beta)\left(l_{2}-9\right)}} d x
\end{aligned}
$$




$$
\begin{aligned}
\leq C\{ & \Lambda_{1} \int_{|x| \leq \varrho_{1}}|x|^{\kappa-N-(\alpha+\beta)\left(l_{2}-9\right)} d x \\
& \left.+\int_{|x|>\varrho_{1}}|Q(x)-Q(0)||x|^{-N-(\alpha+\beta)\left(l_{2}-9\right)} d x\right\} \leq C .
\end{aligned}
$$

So by (14), (16), (19), and the Lebesgue dominated convergence theorem we obtain that

$$
\begin{gathered}
\lim _{\epsilon \rightarrow 0} \epsilon^{-(\alpha+\beta) l_{2}} \int_{\mathbb{R}^{N}}(Q(x)-Q(0))\left[U_{\mu}\left(\frac{|x|}{\epsilon}\right)\right]^{\alpha+\beta} d x \\
=C \int_{\mathbb{R}^{N}}(Q(x)-Q(0))|x|^{-N-(\alpha+\beta)\left(l_{2}-9\right)} d x>0 .
\end{gathered}
$$

Hence, (55) holds for $\epsilon$ sufficiently small.

Part (2), case (i): from (54) it is sufficient to show that

$$
\epsilon^{-(\alpha+\beta) l_{1}} \int_{\mathbb{R}^{N}}(Q(x)-Q(\infty))\left[U_{\mu}\left(\frac{|x|}{\epsilon}\right) d x\right]^{\alpha+\beta} \geq 0,
$$

for some $\epsilon>0$. We choose $R_{1}>0$ such that $Q(x) \geq Q(\infty)+$ $\Lambda_{2}|x|^{-(\alpha+\beta)\left(9-l_{1}\right)}$ for all $|x| \geq R_{1}$. Then

$$
\begin{gathered}
\epsilon^{-(\alpha+\beta) l_{1}} \int_{|x| \geq R_{1}}(Q(x)-Q(\infty))\left[U_{\mu}\left(\frac{|x|}{\epsilon}\right)\right]^{\alpha+\beta} d x \\
=\int_{|x| \geq R_{1}} \frac{Q(x)-Q(\infty)}{|x|^{(\alpha+\beta) l_{1}}}\left[\left(\frac{|x|}{\epsilon}\right)^{l_{1}} U_{\mu}\left(\frac{|x|}{\epsilon}\right)\right]^{\alpha+\beta} d x \\
\quad \geq \Lambda_{2} \int_{|x| \geq R_{1}}|x|^{-N}\left[\left(\frac{|x|}{\epsilon}\right)^{l_{1}} U_{\mu}\left(\frac{|x|}{\epsilon}\right)\right]^{\alpha+\beta} d x \rightarrow+\infty,
\end{gathered}
$$

as $\epsilon \rightarrow \infty$. Moreover, in view of $(\alpha+\beta) l_{1}=N-(\alpha+\beta)\left(\vartheta-l_{1}\right)$ and $(\alpha+\beta)\left(\vartheta-l_{1}\right)>0$, we obtain

$$
\begin{aligned}
& \epsilon^{-(\alpha+\beta) l_{1}} \int_{|x| \leq R_{1}}(Q(x)-Q(\infty))\left[U_{\mu}\left(\frac{|x|}{\epsilon}\right)\right]^{\alpha+\beta} d x \\
& =\int_{|x| \leq R_{1}} \frac{Q(x)-Q(\infty)}{|x|^{(\alpha+\beta) l_{1}}}\left[\left(\frac{|x|}{\epsilon}\right)^{l_{1}} U_{\mu}\left(\frac{|x|}{\epsilon}\right)\right]^{\alpha+\beta} d x \\
& \quad \leq C \int_{|x| \leq R_{1}}|Q(x)-Q(\infty)||x|^{-N+(\alpha+\beta)\left(9-l_{1}\right)} d x \leq \widetilde{C_{2}},
\end{aligned}
$$

for some constant $\widetilde{C_{2}}>0$ independent of $\epsilon>0$. These two estimates combined together give (60) for $\epsilon>0$ large.

Part (2), case (ii): we choose $R_{2}>0$ such that $\mid Q(x)-$ $\left.Q(\infty)\left|\leq \Lambda_{3}\right| x\right|^{-\iota}$ for all $|x| \geq R_{2}$. Since $\iota>(\alpha+\beta)\left(\vartheta-l_{1}\right)>0$ and $(\alpha+\beta) l_{1}=N-(\alpha+\beta)\left(\vartheta-l_{1}\right)$, we get

$$
\begin{aligned}
\int_{\mathbb{R}^{N}}|Q(x)-Q(\infty)||x|^{-N+(\alpha+\beta)\left(9-l_{1}\right)} d x \\
\leq \Lambda_{3} \int_{|x| \geq R_{2}}|x|^{-N-\iota+(\alpha+\beta)\left(9-l_{1}\right)} d x \\
\quad+\int_{|x| \leq R_{2}}|Q(x)-Q(\infty)||x|^{-N+(\alpha+\beta)\left(9-l_{1}\right)} d x<\infty .
\end{aligned}
$$

Consequently, by (13), (16), (21), and the Lebesgue dominated convergence theorem, we have

$$
\begin{gathered}
\lim _{\epsilon \rightarrow \infty} \epsilon^{-(\alpha+\beta) l_{1}} \int_{\mathbb{R}^{N}}(Q(x)-Q(\infty))\left[U_{\mu}\left(\frac{|x|}{\epsilon}\right)\right]^{\alpha+\beta} d x \\
=\lim _{\epsilon \rightarrow \infty} \int_{\mathbb{R}^{N}} \frac{Q(x)-Q(\infty)}{|x|^{(\alpha+\beta) l_{1}}}\left[\left(\frac{|x|}{\epsilon}\right)^{l_{1}} U_{\mu}\left(\frac{|x|}{\epsilon}\right)\right]^{\alpha+\beta} d x \\
=C \int_{\mathbb{R}^{N}}(Q(x)-Q(\infty))|x|^{-N+(\alpha+\beta)\left(\vartheta-l_{1}\right)} d x>0,
\end{gathered}
$$

and (60) holds for $\epsilon>0$ large. Similarly as above, we know part (3) holds.

To prove Theorem 3 we need the following version of the symmetric mountain pass theorem (cf. [31, Theorem 9.12]).

Lemma 12. Let $X$ be an infinite dimensional Banach space and let $\mathscr{E} \in \mathscr{C}^{1}(X, \mathbb{R})$ be an even functional satisfying $(P S)_{c}$ condition for each $c$ and $\mathscr{E}(0)=0$. Furthermore, one supposes that

(i) there exist constants $\bar{\alpha}>0$ and $\rho>0$ such that $\mathscr{E}(w) \geq$ $\bar{\alpha}$ for all $\|w\|=\rho$;

(ii) there exists an increasing sequence of subspaces $\left\{X_{m}\right\}$ of $X$, with $\operatorname{dim} X_{m}=m$, such that for every $m$ one can find a constant $R_{m}>0$ such that $\mathscr{E}(w) \leq 0$ for all $w \in X_{m}$ with $\|w\| \geq R_{m}$.

Then $\mathscr{E}$ possesses a sequence of critical values $\left\{c_{m}\right\}$ tending to $\infty$ as $m \rightarrow \infty$.

Proof of Theorem 3. We follow the arguments of [12]. Applying Lemma 12 with $X=\mathscr{D}_{G}^{1, p}\left(\mathbb{R}^{N}\right) \times \mathscr{D}_{G}^{1, p}\left(\mathbb{R}^{N}\right)$ and $(u, v)=$ $w \in X$, we see from (q.1), (23), and (25) that

$$
\mathscr{E}(u, v) \geq \frac{1}{p}\|(u, v)\|_{\mu}^{p}-\frac{1}{p^{*}}\|Q\|_{\infty}\left(\mathscr{A}_{\mu, \varsigma}^{(\alpha, \beta)}\right)^{-(\alpha+\beta) / p}\|(u, v)\|_{\mu}^{\alpha+\beta}
$$

Since $\alpha+\beta=p^{*}>p$, there exist constants $\bar{\alpha}>0$ and $\rho>0$ such that $\mathscr{E}(u, v) \geq \bar{\alpha}$ for all $(u, v)$ with $\|(u, v)\|_{\mu}=\rho$. To find a suitable sequence of finite dimensional subspaces of $\mathscr{D}_{G}^{1, p}\left(\mathbb{R}^{N}\right) \times \mathscr{D}_{G}^{1, p}\left(\mathbb{R}^{N}\right)$, we set $\Omega=\left\{x \in \mathbb{R}^{N} ; Q(x)>\right.$ $0\}$. Obviously, the set $\Omega$ is $G$-symmetric and we can define $\mathscr{D}_{G}^{1, p}(\Omega) \times \mathscr{D}_{G}^{1, p}(\Omega)$, which is the subspace of $G$-symmetric functions of $\mathscr{D}^{1, p}(\Omega) \times \mathscr{D}^{1, p}(\Omega)$ (see Section 2 ). By extending functions in $\mathscr{D}_{G}^{1, p}(\Omega) \times \mathscr{D}_{G}^{1, p}(\Omega)$ by 0 outside $\Omega$ we can assume that $\mathscr{D}_{G}^{1, p}(\Omega) \times \mathscr{D}_{G}^{1, p}(\Omega) \subset \mathscr{D}_{G}^{1, p}\left(\mathbb{R}^{N}\right) \times \mathscr{D}_{G}^{1, p}\left(\mathbb{R}^{N}\right)$. Let $\left\{X_{m}\right\}$ be an increasing sequence of subspaces of $\mathscr{D}_{G}^{1, p}(\Omega) \times \mathscr{D}_{G}^{1, p}(\Omega)$ with $\operatorname{dim} X_{m}=m$ for each $m$. Then we deduce that there exists a constant $\sigma(m)>0$ such that

$$
\int_{\Omega} Q(x)\left(|\widetilde{u}|^{p^{*}}+|\widetilde{v}|^{p^{*}}+\left.\varsigma \widetilde{u}\right|^{\alpha}|\widetilde{v}|^{\beta}\right) d x \geq \sigma(m),
$$


for all $(\tilde{u}, \widetilde{v}) \in X_{m}$, with $\|(\tilde{u}, \widetilde{v})\|_{\mu}=1$. Consequently, if $(u, v) \epsilon$ $X_{m} \backslash\{(0,0)\}$ then we write $(u, v)=t(\widetilde{u}, \widetilde{v})$, with $t=\|(u, v)\|_{\mu}$ and $\|(\widetilde{u}, \widetilde{v})\|_{\mu}=1$. Hence we obtain

$$
\begin{aligned}
\mathscr{E}(u, v) & =\frac{1}{p} t^{p}-\frac{1}{p^{*}} t^{p^{*}} \int_{\Omega} Q(x)\left(|\widetilde{u}|^{p^{*}}+|\widetilde{v}|^{p^{*}}+\varsigma|\widetilde{u}|^{\alpha}|\widetilde{v}|^{\beta}\right) d x \\
& \leq \frac{1}{p} t^{p}-\frac{\sigma(m)}{p^{*}} t^{p^{*}} \leq 0,
\end{aligned}
$$

for $t$ large enough. Therefore we conclude from Lemma 12 and Corollary 11 that there exists a sequence of critical values $c_{m} \rightarrow \infty$ and the results follow.

Proof of Corollary 4. Since $Q(x)$ is radially symmetric, that is, $Q(x)=Q(|x|)$, we easily see that the corresponding group $G=O(\mathbb{N})$ and $|G|=+\infty$. According to Corollary 11, $\mathscr{E}$ satisfies the $(P S)_{c}$ condition for every $c \in \mathbb{R}$. Hence, by applying the proof of Theorem 3 the conclusion follows.

\section{Multiplicity Results for Problem $\left(\underset{\lambda}{\mathscr{P}_{\lambda}}\right)$}

Throughout this section we assume that $\lambda>0$ and $Q(x) \equiv$ $\widetilde{Q}>0$ is a constant. Since we are interested in positive $G$ symmetric solutions of problem $\left(\mathscr{P}_{\lambda}^{\widetilde{\mathrm{Q}}}\right)$, we define a functional $\mathscr{F}_{\lambda}: \mathscr{D}_{G}^{1, p}\left(\mathbb{R}^{N}\right) \times \mathscr{D}_{G}^{1, p}\left(\mathbb{R}^{N}\right) \rightarrow \mathbb{R}$ given by

$$
\begin{aligned}
\mathscr{F}_{\lambda}(u, v)= & \frac{1}{p}\|(u, v)\|_{\mu}^{p} \\
& -\frac{\widetilde{Q}}{p^{*}} \int_{\mathbb{R}^{N}}\left(\left|u^{+}\right|^{p^{*}}+\left|v^{+}\right|^{p^{*}}+\varsigma\left|u^{+}\right|^{\alpha}\left|v^{+}\right|^{\beta}\right) d x \\
& -\frac{\lambda}{q} \int_{\mathbb{R}^{N}}\left(h_{1}(x)\left|u^{+}\right|^{q}+h_{2}(x)\right)\left|v^{+}\right|^{q} d x
\end{aligned}
$$

where $1<q<p<N, u^{+}=\max \{0, u\}$ and $v^{+}=\max \{0, v\}$. By (h.1), (h.2), and the Hölder inequality, we easily see that $\mathscr{F}_{\lambda} \in \mathscr{C}^{1}\left(\mathscr{D}_{G}^{1, p}\left(\mathbb{R}^{N}\right) \times \mathscr{D}_{G}^{1, p}\left(\mathbb{R}^{N}\right), \mathbb{R}\right)$ and there exists a oneto-one correspondence between the weak solutions of $\left(\mathscr{P}_{\lambda}^{\widetilde{Q}}\right)$ and the critical points of $\mathscr{F}_{\lambda}$. Furthermore, an analogously symmetric principle of Lemma 7 clearly holds; hence, the weak solutions of problem $\left(\mathscr{P}_{\lambda}^{\widetilde{Q}}\right)$ are exactly the critical points of the functional $\mathscr{F}_{\lambda}$.

Lemma 13. Suppose that (h.1) and (h.2) hold. Then there exists a positive constant $M$ depending on $N, p, q, \mathscr{A}_{\mu},\left\|h_{1}\right\|_{\theta}$, and $\left\|h_{2}\right\|_{\theta}$, such that any bounded sequence $\left\{\left(u_{n}, v_{n}\right)\right\} \subset \mathscr{D}_{G}^{1, p}\left(\mathbb{R}^{N}\right) \times$ $\mathscr{D}_{G}^{1, p}\left(\mathbb{R}^{N}\right)$, satisfying

$$
\begin{aligned}
& \mathscr{F}_{\lambda}\left(u_{n}, v_{v}\right) \longrightarrow c<\frac{1}{N} \widetilde{Q}^{(p-N) / p}\left(\mathscr{A}_{\mu, \varsigma}^{(\alpha, \beta)}\right)^{N / p}-M \lambda^{p /(p-q)}, \\
& \mathscr{F}_{\lambda}^{\prime}\left(u_{n}, v_{v}\right) \longrightarrow 0(n \longrightarrow \infty),
\end{aligned}
$$

contains a convergent subsequence.
Proof. Since $\left\{\left(u_{n}, v_{n}\right)\right\}$ is bounded in $\mathscr{D}_{G}^{1, p}\left(\mathbb{R}^{N}\right) \times \mathscr{D}_{G}^{1, p}\left(\mathbb{R}^{N}\right)$, we can obtain a subsequence, still denoted by $\left\{\left(u_{n}, v_{n}\right)\right\}$, satisfying

$$
\begin{aligned}
& u_{n} \rightarrow u, \quad v_{n} \rightarrow v, \quad \text { in } \mathscr{D}_{G}^{1, p}\left(\mathbb{R}^{N}\right), \\
& u_{n} \longrightarrow u, \quad v_{n} \longrightarrow v, \quad \text { a.e. in } \mathbb{R}^{N}, \\
& \nabla u_{n} \longrightarrow \nabla u, \quad \nabla v_{n} \longrightarrow \nabla v, \quad \text { a.e. in } \mathbb{R}^{N} .
\end{aligned}
$$

Moreover, using (h.2) and the Hölder inequality and the Lebesgue dominated theorem, we may also assume

$$
\begin{aligned}
& \int_{\mathbb{R}^{N}} h_{1}(x)\left|u_{n}^{+}\right|^{q} d x \longrightarrow \int_{\mathbb{R}^{N}} h_{1}(x)\left|u^{+}\right|^{q} d x, \\
& \int_{\mathbb{R}^{N}} h_{2}(x)\left|v_{n}^{+}\right|^{q} d x \longrightarrow \int_{\mathbb{R}^{N}} h_{2}(x)\left|v^{+}\right|^{q} d x,
\end{aligned}
$$

as $n \rightarrow \infty$. By (71) and the standard argument, we easily show that $(u, v)$ is a critical point of $\mathscr{F}_{\lambda}$. Consequently, we deduce from (68), (9), (h.2), the Hölder inequality, and the fact that $1<q<p<p^{*}$ that

$$
\begin{aligned}
\mathscr{F}_{\lambda}(u, v)= & \frac{1}{N}\left\|\left(u_{n}, v_{n}\right)\right\|_{\mu}^{p}-\left(\frac{1}{q}-\frac{1}{p^{*}}\right) \lambda \\
& \times \int_{\mathbb{R}^{N}}\left(h_{1}(x)\left|u^{+}\right|^{q}+h_{2}(x)\left|v^{+}\right|^{q}\right) d x \\
\geq & \frac{1}{N}\|(u, v)\|_{\mu}^{p}-\frac{p^{*}-q}{q p^{*}} \mathscr{A}_{\mu}^{-q / p} \lambda \\
& \times\left(\left\|h_{1}\right\|_{\theta}\|u\|_{\mu}^{q}+\left\|h_{2}\right\|_{\theta}\|v\|_{\mu}^{q}\right) \\
\geq & \frac{1}{N}\left(\|u\|_{\mu}^{p}+\|v\|_{\mu}^{p}\right)-\frac{p^{*}-q}{q p^{*}} \mathscr{A}_{\mu}^{-q / p} \lambda \\
& \times \max \left\{\left\|h_{1}\right\|_{\theta},\left\|h_{2}\right\|_{\theta}\right\}\left(\|u\|_{\mu}^{q}+\|v\|_{\mu}^{q}\right) \\
\geq & -\frac{2(p-q)}{p}\left(\frac{q N}{p}\right)^{q /(p-q)} \\
& \times\left[\frac{\lambda^{*}-q}{q p^{*}} \mathscr{A}_{\mu}^{-q / p} \max _{\mu}\left\{\left\|h_{1}\right\|_{\theta},\left\|h_{2}\right\|_{\theta}\right\}\right]^{p /(p-q)} \\
& {\left[M \lambda^{p /(p-q)},\right.}
\end{aligned}
$$

where $M=(2(p-q) / p)(q N / p)^{q /(p-q)}\left[\left(\left(p^{*}-q\right) /\right.\right.$ $\left.\left.\left.q p^{*}\right) \mathscr{A}_{\mu}^{-q / p} \max \left\{\left\|h_{1}\right\|_{\theta},\left\|h_{2}\right\|_{\theta}\right\}\right]^{p /(p-q)}\right)$ is a positive constant. 
Now we set $\bar{u}_{n}=u_{n}-u$ and $\bar{v}_{n}=v_{n}-v$. Then by the BrezisLieb lemma [32] and arguing as in [33, Lemma 2.1] we get

$$
\begin{aligned}
\left\|\left(\bar{u}_{n}, \bar{v}_{n}\right)\right\|_{\mu}^{p} & =\left\|\left(u_{n}, v_{n}\right)\right\|_{\mu}^{p}-\|(u, v)\|_{\mu}^{p}+o_{n}(1), \\
\int_{\mathbb{R}^{N}}\left|\bar{u}_{n}^{+}\right|^{p^{*}} d x= & \int_{\mathbb{R}^{N}}\left|u_{n}^{+}\right|^{p^{*}} d x-\int_{\mathbb{R}^{N}}\left|u^{+}\right|^{p^{*}} d x+o_{n}(1), \\
\int_{\mathbb{R}^{N}}\left|\bar{v}_{n}^{+}\right|^{p^{*}} d x= & \int_{\mathbb{R}^{N}}\left|v_{n}^{+}\right|^{p^{*}} d x-\int_{\mathbb{R}^{N}}\left|v^{+}\right|^{p^{*}} d x+o_{n}(1), \\
\int_{\mathbb{R}^{N}}\left|\bar{u}_{n}^{+}\right|^{\alpha}\left|\bar{v}_{n}^{+}\right|^{\beta} d x= & \int_{\mathbb{R}^{N}}\left|u_{n}^{+}\right|^{\alpha}\left|v_{n}^{+}\right|^{\beta} d x \\
& -\int_{\mathbb{R}^{N}}\left|u^{+}\right|^{\alpha}\left|v^{+}\right|^{\beta} d x+o_{n}(1) .
\end{aligned}
$$

Since $\mathscr{F}_{\lambda}\left(u_{n}, v_{n}\right)=c+o_{n}(1)$ and $\mathscr{F}_{\lambda}^{\prime}\left(u_{n}, v_{n}\right)=o_{n}(1)$, we obtain from (68), (71), and (73) that

$$
\begin{aligned}
& \frac{1}{p}\left\|\left(\bar{u}_{n}, \bar{v}_{n}\right)\right\|_{\mu}^{p}-\frac{\widetilde{Q}}{p^{*}} \int_{\mathbb{R}^{N}}\left(\left|\bar{u}_{n}^{+}\right|^{p^{*}}+\left|\bar{v}_{n}^{+}\right|^{p^{*}}+\varsigma\left|\bar{u}_{n}^{+}\right|^{\alpha}\left|\bar{v}_{n}^{+}\right|^{\beta}\right) d x \\
& \quad=c-\mathscr{F}_{\lambda}(u, v)+o_{n}(1), \\
& \left\|\left(\bar{u}_{n}, \bar{v}_{n}\right)\right\|_{\mu}^{p}-\widetilde{Q} \int_{\mathbb{R}^{N}}\left(\left|\bar{u}_{n}^{+}\right|^{p^{*}}+\left|\bar{v}_{n}^{+}\right|^{p^{*}}+\varsigma\left|\bar{u}_{n}^{+}\right|^{\alpha}\left|\bar{v}_{n}^{+}\right|^{\beta}\right) d x \\
& \quad=o_{n}(1) .
\end{aligned}
$$

Hence, for a subsequence $\left\{\left(\bar{u}_{n}, \bar{v}_{n}\right)\right\}$, we have

$$
\begin{gathered}
\left\|\left(\bar{u}_{n}, \bar{v}_{n}\right)\right\|_{\mu}^{p} \longrightarrow k \geq 0, \\
\widetilde{Q} \int_{\mathbb{R}^{N}}\left(\left|\bar{u}_{n}^{+}\right|^{p^{*}}+\left|\bar{v}_{n}^{+}\right|^{p^{*}}+\varsigma\left|\bar{u}_{n}^{+}\right|^{\alpha}\left|\bar{v}_{n}^{+}\right|^{\beta}\right) d x \longrightarrow k,
\end{gathered}
$$

as $n \rightarrow \infty$. From the definition (25) of $\mathscr{A}_{\mu, \varsigma}^{(\alpha, \beta)}$ it follows that $\mathscr{A}_{\mu, \varsigma}^{(\alpha, \beta)}(k / \widetilde{Q})^{p /(\alpha+\beta)} \leq k$, which implies either $k=0$ or $k \geq \widetilde{Q}^{(p-N) / p}\left(\mathscr{A}_{\mu, \zeta}^{(\alpha, \beta)}\right)^{N / p}$. If $k \geq \widetilde{Q}^{(p-N) / p}\left(\mathscr{A}_{\mu, \zeta}^{(\alpha, \beta)}\right)^{N / p}$, we obtain from (72) and (74) that

$$
\begin{aligned}
c= & \mathscr{F}_{\lambda}(u, v)+\left(\frac{1}{p}-\frac{1}{p^{*}}\right) k \geq \frac{1}{N} \widetilde{Q}^{(p-N) / p}\left(\mathscr{A}_{\mu, \varsigma}^{(\alpha, \beta)}\right)^{N / p} \\
& -M \lambda^{p /(p-q)}
\end{aligned}
$$

which contradicts (69). Consequently, we have $\left\|\left(\bar{u}_{n}, \bar{v}_{n}\right)\right\|_{\mu}^{p} \rightarrow$ 0 as $n \rightarrow \infty$, and, thus, $\left(u_{n}, v_{n}\right) \rightarrow(u, v)$ in $\mathscr{D}_{G}^{1, p}\left(\mathbb{R}^{N}\right) \times$ $\mathscr{D}_{G}^{1, p}\left(\mathbb{R}^{N}\right)$. The lemma is proved.

Lemma 14. Suppose that (h.1) and (h.2) hold. Then there exists $\lambda_{1}^{*}>0$ such that for any $\lambda \in\left(0, \lambda_{1}^{*}\right)$ the following geometric conditions for $\mathscr{F}_{\lambda}(u, v)$ hold:

(i) $\mathscr{F}_{\lambda}(0,0)=0$; there exist $\tilde{\alpha}>0$ and $\rho>0$ such that $\mathscr{F}_{\lambda}(u, v) \geq \tilde{\alpha}$ for all $\|(u, v)\|_{\mu}=\rho$; (ii) there exists $\left(e_{u}, e_{v}\right) \in \mathscr{D}_{G}^{1, p}\left(\mathbb{R}^{N}\right) \times \mathscr{D}_{G}^{1, p}\left(\mathbb{R}^{N}\right)$ such that $\left\|\left(e_{u}, e_{v}\right)\right\|_{\mu}>\rho$ and $\mathscr{F}_{\lambda}\left(e_{u}, e_{v}\right)<0$.

Proof. According to (h.1) and (h.2), for all $0<\sigma<1 / p$, we deduce from (9), (25), (68), the Young inequality, and the Hölder inequality that

$$
\begin{aligned}
\mathscr{F}_{\lambda}(u, v) \geq & \frac{1}{p}\|(u, v)\|_{\mu}^{p}-\frac{1}{p^{*}} \widetilde{Q}\left(\mathscr{A}_{\mu, \varsigma}^{(\alpha, \beta)}\right)^{-(\alpha+\beta) / p}\|(u, v)\|_{\mu}^{\alpha+\beta} \\
& -\frac{\lambda}{q} \mathscr{A}_{\mu}^{-q / p} \max \left\{\left\|h_{1}\right\|_{\theta},\left\|h_{2}\right\|_{\theta}\right\}\left(\|u\|_{\mu}^{q}+\|v\|_{\mu}^{q}\right) \\
\geq & \frac{1}{p}\|(u, v)\|_{\mu}^{p}-\frac{1}{p^{*}} \widetilde{Q}\left(\mathscr{A}_{\mu, \varsigma}^{(\alpha, \beta)}\right)^{-(\alpha+\beta) / p} \\
& \times\|(u, v)\|_{\mu}^{\alpha+\beta}-C \lambda\|(u, v)\|_{\mu}^{q} \\
\geq & \left(\frac{1}{p}-\sigma\right)\|(u, v)\|_{\mu}^{p}-\frac{1}{p^{*}} \widetilde{Q}\left(\mathscr{A}_{\mu, \varsigma}^{(\alpha, \beta)}\right)^{-(\alpha+\beta) / p} \\
& \times\|(u, v)\|_{\mu}^{\alpha+\beta}-C(\sigma) \lambda^{p /(p-q)},
\end{aligned}
$$

where $C(\sigma)>0$ is a constant depending on $\sigma>0$. The last inequality and the fact $\alpha+\beta=p^{*}>p$ imply that, for small $\sigma$, there exist constants $\tilde{\alpha}>0, \rho>0$, and $\lambda_{1}^{*}>0$ such that $\mathscr{F}_{\lambda}(u, v) \geq \tilde{\alpha}>0$ for all $\|(u, v)\|_{\mu}=\rho$ and $0<\lambda<\lambda_{1}^{*}$. On the other hand, since $\int_{\mathbb{R}^{N}}\left(h_{1}(x)\left|u^{+}\right|^{q}+h_{2}(x)\left|v^{+}\right|^{q}\right) d x \geq 0$, we conclude from $(68)$ that there exists $(\widetilde{u}, \widetilde{v}) \in \mathscr{D}_{G}^{1, p}\left(\mathbb{R}^{N}\right) \times$ $\mathscr{D}_{G}^{1, p}\left(\mathbb{R}^{N}\right) \backslash\{(0,0)\}$ such that $\mathscr{F}_{\lambda}(t \tilde{u}, t \widetilde{v}) \rightarrow-\infty$ as $t \rightarrow+\infty$, which completes this proof.

Lemma 15. Suppose that (h.1) and (h.2) hold. Then there exists $\lambda_{2}^{*}>0$ such that

$$
\sup _{t \geq 0} \mathscr{F}_{\lambda}\left(t y_{\epsilon}, t \tau_{\min } y_{\epsilon}\right)<\frac{1}{N} \widetilde{Q}^{(p-N) / p}\left(\mathscr{A}_{\mu, \varsigma}^{(\alpha, \beta)}\right)^{N / p}-M \lambda^{p /(p-q)}
$$

for any $\lambda \in\left(0, \lambda_{2}^{*}\right)$ and small $\epsilon>0$, where $\tau_{\min }>0$ satisfies (26)-(28) and $M>0$ is given in Lemma 13.

Proof. First, we define the functions

$$
\begin{aligned}
\Psi(t)= & \mathscr{F}_{\lambda}\left(t y_{\epsilon}, t \tau_{\min } y_{\epsilon}\right)=\frac{t^{p}}{p}\left(1+\tau_{\text {min }}^{p}\right) \\
& \times \int_{\mathbb{R}^{N}}\left(\left|\nabla y_{\epsilon}\right|^{p}-\mu \frac{\left|y_{\epsilon}\right|^{p}}{|x|^{p}}\right) d x \\
& -\frac{\widetilde{Q} t^{p^{*}}}{p^{*}}\left(1+\varsigma \tau_{\min }^{\beta}+\tau_{\min }^{\alpha+\beta}\right) \int_{\mathbb{R}^{N}}\left|y_{\epsilon}\right|^{p^{*}} d x \\
& -\frac{\lambda t^{q}}{q} \int_{\mathbb{R}^{N}}\left(h_{1}(x)+\tau_{\min }^{q} h_{2}(x)\right)\left|y_{\epsilon}\right|^{q} d x,
\end{aligned}
$$




$$
\begin{aligned}
\widetilde{\Psi}(t)= & \frac{t^{p}}{p}\left(1+\tau_{\min }^{p}\right) \int_{\mathbb{R}^{N}}\left(\left|\nabla y_{\epsilon}\right|^{p}-\mu \frac{\left|y_{\epsilon}\right|^{p}}{|x|^{p}}\right) d x \\
& -\frac{\widetilde{Q} t^{p^{*}}}{p^{*}}\left(1+\varsigma \tau_{\min }^{\beta}+\tau_{\min }^{\alpha+\beta}\right) \int_{\mathbb{R}^{N}}\left|y_{\epsilon}\right|^{p^{*}} d x,
\end{aligned}
$$

with $t \geq 0$. Note that $\widetilde{\Psi}(0)=0, \widetilde{\Psi}(t)>0$ for $t \rightarrow 0^{+}$, and $\lim _{t \rightarrow+\infty} \widetilde{\Psi}(t)=-\infty$. Hence $\sup _{t \geq 0} \widetilde{\Psi}(t)$ can be achieved at some finite $\tilde{t}_{\epsilon}>0$ at which $\widetilde{\Psi}^{\prime}(t)$ becomes zero. By direct calculation, we obtain from (11), (12), (26), (27), (80), and Lemma 8 that

$$
\begin{aligned}
& \sup _{t \geq 0} \widetilde{\Psi}(t) \\
& =\widetilde{\Psi}\left(\widetilde{t}_{\epsilon}\right)=\frac{1}{N} \\
& \quad \times\left\{\frac{\left(1+\tau_{\min }^{p}\right) \int_{\mathbb{R}^{N}}\left(\left|\nabla y_{\epsilon}\right|^{p}-\mu\left(\left|y_{\epsilon}\right|^{p} /|x|^{p}\right)\right) d x}{\left(\widetilde{Q}\left(1+\varsigma \tau_{\min }^{\beta}+\tau_{\min }^{\alpha+\beta}\right) \int_{\mathbb{R}^{N}}\left|y_{\epsilon}\right|^{*} d x\right)^{p / p^{*}}}\right\}^{p^{*} /\left(p^{*}-p\right)} \\
& =\frac{1}{N} \widetilde{Q}^{(p-N) / p}\left(K\left(\tau_{\min }\right) \mathscr{A}_{\mu}\right)^{N / p}=\frac{1}{N} \widetilde{Q}^{(p-N) / p}\left(\mathscr{A}_{\mu, \zeta}^{(\alpha, \beta)}\right)^{N / p} .
\end{aligned}
$$

Let $\bar{\Lambda}>0$ be such that $(1 / N) \widetilde{Q}^{(p-N) / p}\left(\mathscr{A}_{\mu, \varsigma}^{(\alpha, \beta)}\right)^{N / p}-$ $M \lambda^{p /(p-q)}>0, \forall \lambda \in(0, \bar{\Lambda})$. Then from (h.1), (h.2), (11), and (79), we have

$$
\Psi(t)=\mathscr{F}_{\lambda}\left(t y_{\epsilon}, t \tau_{\min } y_{\epsilon}\right) \leq \frac{t^{p}}{p}\left(1+\tau_{\min }^{p}\right), \quad \forall t \geq 0, \lambda>0,
$$

and there exists $T_{0} \in(0,1)$ independent of $\epsilon$ such that

$$
\begin{aligned}
\sup _{0 \leq t \leq T_{0}} \Psi(t) \leq & \frac{T_{0}^{p}}{p}\left(1+\tau_{\min }^{p}\right)<\frac{1}{N} \widetilde{Q}^{(p-N) / p}\left(\mathscr{A}_{\mu, \varsigma}^{(\alpha, \beta)}\right)^{N / p} \\
& -M \lambda^{p /(p-q)}, \quad \forall \lambda \in(0, \bar{\Lambda}) .
\end{aligned}
$$

Moreover, we obtain from (79)-(81) that

$$
\begin{aligned}
\sup _{t \geq T_{0}} \Psi(t) \leq & \sup _{t \geq 0} \widetilde{\Psi}(t) \\
& -\frac{\lambda}{q} T_{0}^{q} \int_{\mathbb{R}^{N}}\left(h_{1}(x)+\tau_{\min }^{q} h_{2}(x)\right)\left|y_{\epsilon}\right|^{q} d x \\
= & \frac{1}{N} \widetilde{Q}^{(p-N) / p}\left(\mathscr{A}_{\mu, \varsigma}^{(\alpha, \beta)}\right)^{N / p} \\
& -\frac{\lambda}{q} T_{0}^{q} \int_{\mathbb{R}^{N}}\left(h_{1}(x)+\tau_{\min }^{q} h_{2}(x)\right)\left|y_{\epsilon}\right|^{q} d x .
\end{aligned}
$$

Now, taking $\lambda>0$ such that

$$
-\frac{\lambda}{q} T_{0}^{q} \int_{\mathbb{R}^{N}}\left(h_{1}(x)+\tau_{\min }^{q} h_{2}(x)\right)\left|y_{\epsilon}\right|^{q} d x<-M \lambda^{p /(p-q)},
$$

that is,

$$
0<\lambda<\left(\frac{T_{0}^{q}}{q M} \int_{\mathbb{R}^{N}}\left(h_{1}(x)+\tau_{\min }^{q} h_{2}(x)\right)\left|y_{\epsilon}\right|^{q} d x\right)^{(p-q) / p} \triangleq \widetilde{\Lambda},
$$

we have

$\sup _{t \geq T_{0}} \Psi(t)<\frac{1}{N} \widetilde{Q}^{(p-N) / p}\left(\mathscr{A}_{\mu, \varsigma}^{(\alpha, \beta)}\right)^{N / p}-M \lambda^{p /(p-q)}, \quad \forall \lambda \in(0, \widetilde{\Lambda})$.

Choosing $\lambda_{2}^{*}=\min \{\bar{\Lambda}, \widetilde{\Lambda}\}$, we deduce from (83) and (87) that

$$
\begin{array}{r}
\sup _{t \geq 0} \Psi(t)<\frac{1}{N} \widetilde{Q}^{(p-N) / p}\left(\mathscr{A}_{\mu, \varsigma}^{(\alpha, \beta)}\right)^{N / p}-M \lambda^{p /(p-q)}, \\
\forall \lambda \in\left(0, \lambda_{2}^{*}\right) .
\end{array}
$$

Therefore the result of this lemma follows.

Proof of Theorem 5. Taking $\rho>0$ and $\lambda^{*}=\min \left\{\lambda_{1}^{*}, \lambda_{2}^{*}\right\}$, for $0<\lambda<\lambda^{*}$, given in the proofs of Lemmas 14 and 15, we define

$$
c_{1} \triangleq \inf _{\bar{B}_{\rho}(0)} \mathscr{F}_{\lambda}(u, v)
$$

where $\bar{B}_{\rho}(0)=\left\{(u, v) \in \mathscr{D}_{G}^{1, p}\left(\mathbb{R}^{N}\right) \times \mathscr{D}_{G}^{1, p}\left(\mathbb{R}^{N}\right) ;\|(u, v)\|_{\mu} \leq\right.$ $\rho$ \}. Since the metric space $\bar{B}_{\rho}(0)$ is complete, we deduce from the Ekeland variational principle [34] that there exists a sequence $\left\{\left(u_{n}, v_{n}\right)\right\} \subset \bar{B}_{\rho}(0)$ such that $\mathscr{F}_{\lambda}\left(u_{n}, v_{n}\right) \rightarrow c_{1}$ and $\mathscr{F}_{\lambda}^{\prime}\left(u_{n}, v_{n}\right) \rightarrow 0$ as $n \rightarrow \infty$. Let $\varphi_{0}, \psi_{0} \in \mathscr{C}_{0}^{\infty}\left(\mathbb{R}^{N}\right)$ be the $G$-symmetric functions such that $\varphi_{0}, \psi_{0}>0$. By (h.1) and (h.2), we have $\int_{\mathbb{R}^{N}}\left(h_{1}(x) \varphi_{0}^{q}+h_{2}(x) \psi_{0}^{q}\right) d x>0$. This, combined with the fact that $1<q<p<p^{*}$, implies that there exists $t_{0}=t_{0}\left(\varphi_{0}, \psi_{0}\right)>0$ sufficiently small such that

$$
\begin{aligned}
\mathscr{F}_{\lambda}\left(t_{0} \varphi_{0}, t_{0} \psi_{0}\right)= & \frac{t_{0}^{p}}{p}\left\|\left(\varphi_{0}, \psi_{0}\right)\right\|_{\mu}^{p} \\
& -\frac{\widetilde{Q}}{p^{*}} t_{0}^{p^{*}} \int_{\mathbb{R}^{N}}\left(\varphi_{0}^{p^{*}}+\psi_{0}^{p^{*}}+\varsigma \varphi_{0}^{\alpha} \psi_{0}^{\beta}\right) d x \\
& -\frac{\lambda}{q} t_{0}^{q} \int_{\mathbb{R}^{N}}\left(h_{1}(x) \varphi_{0}^{q}+h_{2}(x) \psi_{0}^{q}\right) d x<0 .
\end{aligned}
$$

Therefore we obtain $c_{1}<0<(1 / N) \widetilde{Q}^{(p-N) / p}\left(\mathscr{A}_{\mu, \zeta}^{(\alpha, \beta)}\right)^{N / p}-$ $M \lambda^{p /(p-q)}$ for any $\lambda \in\left(0, \lambda^{*}\right)$. By Lemma $13, \mathscr{F}_{\lambda}$ possesses a critical point $\left(u_{1}, v_{1}\right)$ with $\mathscr{F}_{\lambda}\left(u_{1}, v_{1}\right)=c_{1}<0$. Taking $\left(u_{1}^{-}, v_{1}^{-}\right)$as a pair of test functions, where $u_{1}^{-}=\min \left\{0, u_{1}\right\}$ and $v_{1}^{-}=\min \left\{0, v_{1}\right\}$, we deduce from (68) that $0=$ $\left\langle\mathscr{F}_{\lambda}^{\prime}\left(u_{1}, v_{1}\right),\left(u_{1}^{-}, v_{1}^{-}\right)\right\rangle=\left\|\left(u_{1}^{-}, v_{1}^{-}\right)\right\|_{\mu}^{p}$, which implies $u_{1} \geq 0$ and $v_{1} \geq 0$ in $\mathbb{R}^{N}$. Consequently, by the strong maximum principle and the symmetric criticality principle, we conclude that $\left(u_{1}, v_{1}\right)$ is a positive $G$-symmetric solution of problem $\left(\mathscr{P}_{\lambda}^{\widetilde{Q}}\right)$. 
On the other hand, we define

$$
c_{2} \triangleq \inf _{\gamma \in \Gamma \in[0,1]} \max _{\lambda} \mathscr{F}_{\lambda}(\gamma(t))
$$

where $\Gamma=\left\{\gamma \in \mathscr{C}\left([0,1], \mathscr{D}_{G}^{1, p}\left(\mathbb{R}^{N}\right) \times \mathscr{D}_{G}^{1, p}\left(\mathbb{R}^{N}\right)\right) ; \gamma(0)=\right.$ $\left.(0,0), \gamma(1)=\left(e_{u}, e_{v}\right)\right\}$. It follows from Lemmas 14 and 15 that

$$
\begin{aligned}
0<\tilde{\alpha} \leq c_{2}<\frac{1}{N} \widetilde{Q}^{(p-N) / p}\left(\mathscr{A}_{\mu, \varsigma}^{(\alpha, \beta)}\right)^{N / p} & -M \lambda^{p /(p-q),} \\
& \forall \lambda \in\left(0, \lambda^{*}\right) .
\end{aligned}
$$

Hence $c_{2}$ is a critical value of $\mathscr{F}_{\lambda}$ by the mountain pass theorem. Similar to the arguments above, problem $\left(\mathscr{P}_{\lambda}^{\widetilde{Q}}\right)$ admits another positive $G$-symmetric solution $\left(u_{2}, v_{2}\right)$ with $\mathscr{F}_{\lambda}\left(u_{2}, v_{2}\right)=c_{2}>0$.

\section{Conflict of Interests}

The authors declare that there is no conflict of interests regarding the publication of this paper.

\section{Acknowledgments}

The project is supported by the Natural Science Foundation of China (Grants nos. 11471235 and 11171247), and it is supported by the Scientific and Technological Research Program of Chongqing Municipal Education Commission (Grant no. KJ130503).

\section{References}

[1] R. Dautray and J. L. Lions, "Mathematical analysis and numerical methods for science and technology," in Physical Origins and Classical Methods, Springer, Berlin, Germany, 1990.

[2] J. P. García Azorero and I. Peral Alonso, "Hardy inequalities and some critical elliptic and parabolic problems," Journal of Differential Equations, vol. 144, no. 2, pp. 441-476, 1998.

[3] N. Ghoussoub and C. Yuan, "Multiple solutions for quasi-linear PDEs involving the critical Sobolev and Hardy exponents," Transactions of the American Mathematical Society, vol. 352, no. 12 , pp. 5703-5743, 2000.

[4] D. Kang, "On the quasilinear elliptic problems with critical Sobolev-HARdy exponents and HARdy terms," Nonlinear Analysis: Theory, Methods \& Applications, vol. 68, no. 7, pp. 19731985, 2008.

[5] Z. Chen and W. Zou, "On an elliptic problem with critical exponent and Hardy potential," Journal of Differential Equations, vol. 252, no. 2, pp. 969-987, 2012.

[6] M. Ramaswamy and S. Santra, "Uniqueness and profile of positive solutions of a critical exponent problem with Hardy potential," Journal of Differential Equations, vol. 254, no. 11, pp. 4347-4372, 2013.

[7] H. Liu and L. Zhao, "Nontrivial solutions for a class of critical elliptic equations of Caffarelli-Kohn-Nirenberg type," Journal of Mathematical Analysis and Applications, vol. 404, no. 2, pp. 317325, 2013.

[8] P. C. Carrião, R. Demarque, and O. H. Miyagaki, "Existence and non-existence of solutions for $\mathrm{p}$-Laplacian equations with decaying cylindrical potentials," Journal of Differential Equations, vol. 255, no. 10, pp. 3412-3433, 2013.
[9] Y. Deng and L. Jin, "On symmetric solutions of a singular elliptic equation with critical Sobolev-Hardy exponent," Journal of Mathematical Analysis and Applications, vol. 329, no. 1, pp. 603-616, 2007.

[10] S. Waliullah, "Minimizers and symmetric minimizers for problems with critical Sobolev exponent," Topological Methods in Nonlinear Analysis, vol. 34, no. 2, pp. 291-326, 2009.

[11] Z. Deng and Y. Huang, "On G-symmetric solutions of a quasilinear elliptic equation involving critical Hardy-Sobolev exponent," Journal of Mathematical Analysis and Applications, vol. 384, no. 2, pp. 578-590, 2011.

[12] G. Bianchi, J. Chabrowski, and A. Szulkin, "On symmetric solutions of an elliptic equation with a nonlinearity involving critical Sobolev exponent," Nonlinear Analysis: Theory, Methods \& Applications, vol. 25, no. 1, pp. 41-59, 1995.

[13] T. Bartsch and M. Willem, Infinitely Many Non-Radial Solutions of an Euclidean Scalar Field Equation, Mathematisches Institut, Universitat Heidelberg, Heidelberg, Germany, 1992.

[14] J. Chabrowski, "On the existence of G-symmetric entire solutions for semilinear elliptic equations," Rendiconti del Circolo Matematico di Palermo, vol. 41, no. 3, pp. 413-440, 1992.

[15] T.-F. Wu, "The Nehari manifold for a semilinear elliptic system involving sign-changing weight functions," Nonlinear Analysis: Theory, Methods \& Applications, vol. 68, no. 6, pp. 1733-1745, 2008.

[16] N. Nyamoradi, "Existence and multiplicity of solutions to a singular elliptic system with critical Sobolev-Hardy exponents and concave-convex nonlinearities," Journal of Mathematical Analysis and Applications, vol. 396, no. 1, pp. 280-293, 2012.

[17] D. Lü and J. Xiao, "Multiple solutions for weighted nonlinear elliptic system involving critical exponents," Mathematical and Computer Modelling, vol. 55, no. 3-4, pp. 816-827, 2012.

[18] Y. Li and W. Gao, "Existence of multiple solutions for singular quasilinear elliptic system with critical sobolev-hardy exponents and concave-convex terms," Acta Mathematica Scientia B, vol. 33, no. 1, pp. 107-121, 2013.

[19] C. O. Alves, D. C. de Morais Filho, and M. A. S. Souto, "On systems of elliptic equations involving subcritical or critical Sobolev exponents," Nonlinear Analysis, vol. 42, no. 5, pp. 771$787,2000$.

[20] J. Long and J. Yang, "Existence results for critical singular elliptic systems," Nonlinear Analysis: Theory, Methods \& Applications, vol. 69, no. 11, pp. 4199-4214, 2008.

[21] M. de Souza, "On a singular class of elliptic systems involving critical growth in $\mathbb{R}^{2}$," Nonlinear Analysis: Real World Applications, vol. 12, no. 2, pp. 1072-1088, 2011.

[22] S. Benmouloud, R. Echarghaoui, and S. M. Sbaï, "Multiplicity of positive solutions for a critical quasilinear elliptic system with concave and convex nonlinearities," Journal of Mathematical Analysis and Applications, vol. 396, no. 1, pp. 375-385, 2012.

[23] J. Sun, H. Chen, and J. J. Nieto, "On ground state solutions for some non-autonomous Schrödinger-Poisson systems," Journal of Differential Equations, vol. 252, no. 5, pp. 3365-3380, 2012.

[24] Z. Deng and Y. Huang, "Existence of symmetric solutions for singular semilinear elliptic systems with critical Hardy-Sobolev exponents," Nonlinear Analysis: Real World Applications, vol. 14, no. 1, pp. 613-625, 2013.

[25] D. Huang and Y. Li, "Multiplicity of solutions for a noncooperative $p$-Laplacian elliptic system in $\mathbb{R}^{N}$," Journal of Differential Equations, vol. 215, no. 1, pp. 206-223, 2005. 
[26] R. S. Palais, “The principle of symmetric criticality," Communications in Mathematical Physics, vol. 69, no. 1, pp. 19-30, 1979.

[27] P. L. Lions, "The concentration-compactness principle in the calculus of variations," Revista Matemática Iberoamericana, vol. 1, no. 1, part 1, pp. 145-201, 1985.

[28] P. Lions, "The concentration-compactness principle in the calculus of variations. The limit case, part 2," Revista Matemática Iberoamericana, vol. 1, no. 2, pp. 45-121, 1985.

[29] A. Ambrosetti and P. H. Rabinowitz, "Dual variational methods in critical point theory and applications," Journal of Functional Analysis, vol. 14, pp. 349-381, 1973.

[30] H. Brezis and L. Nirenberg, "Positive solutions of nonlinear elliptic equations involving critical Sobolev exponents," Communications on Pure and Applied Mathematics, vol. 36, no. 4, pp. 437-477, 1983.

[31] H. Rabinowitz, Methods in Critical Point Theory with Applications to Differential Equations, CBMS Regional Conference Series in Mathematics, American Mathematical Society, 1986.

[32] H. Brézis and E. Lieb, "A relation between pointwise convergence of functions and convergence of functionals," Proceedings of the American Mathematical Society, vol. 88, no. 3, pp. 486490, 1983.

[33] P. Han, "The effect of the domain topology on the number of positive solutions of an elliptic system involving critical Sobolev exponents," Houston Journal of Mathematics, vol. 32, no. 4, pp. 1241-1257, 2006.

[34] I. Ekeland, "Nonconvex minimization problems," Bulletin of the American Mathematical Society, vol. 1, no. 3, pp. 443-474, 1979. 


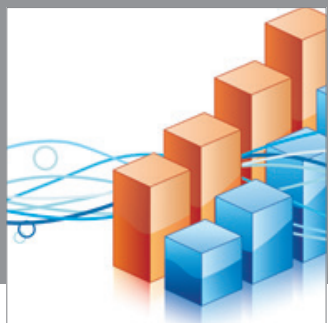

Advances in

Operations Research

mansans

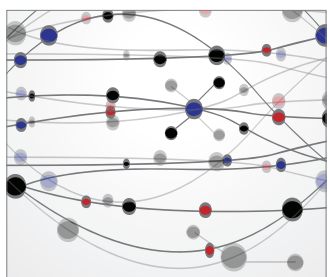

The Scientific World Journal
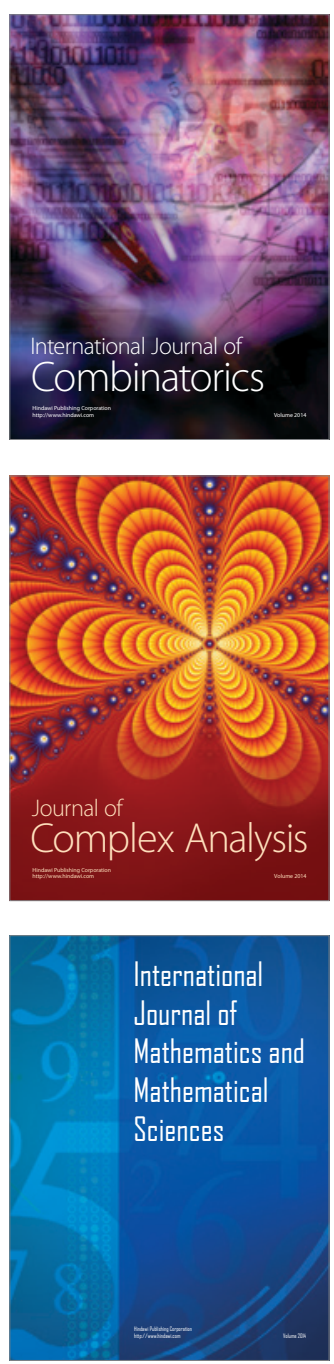
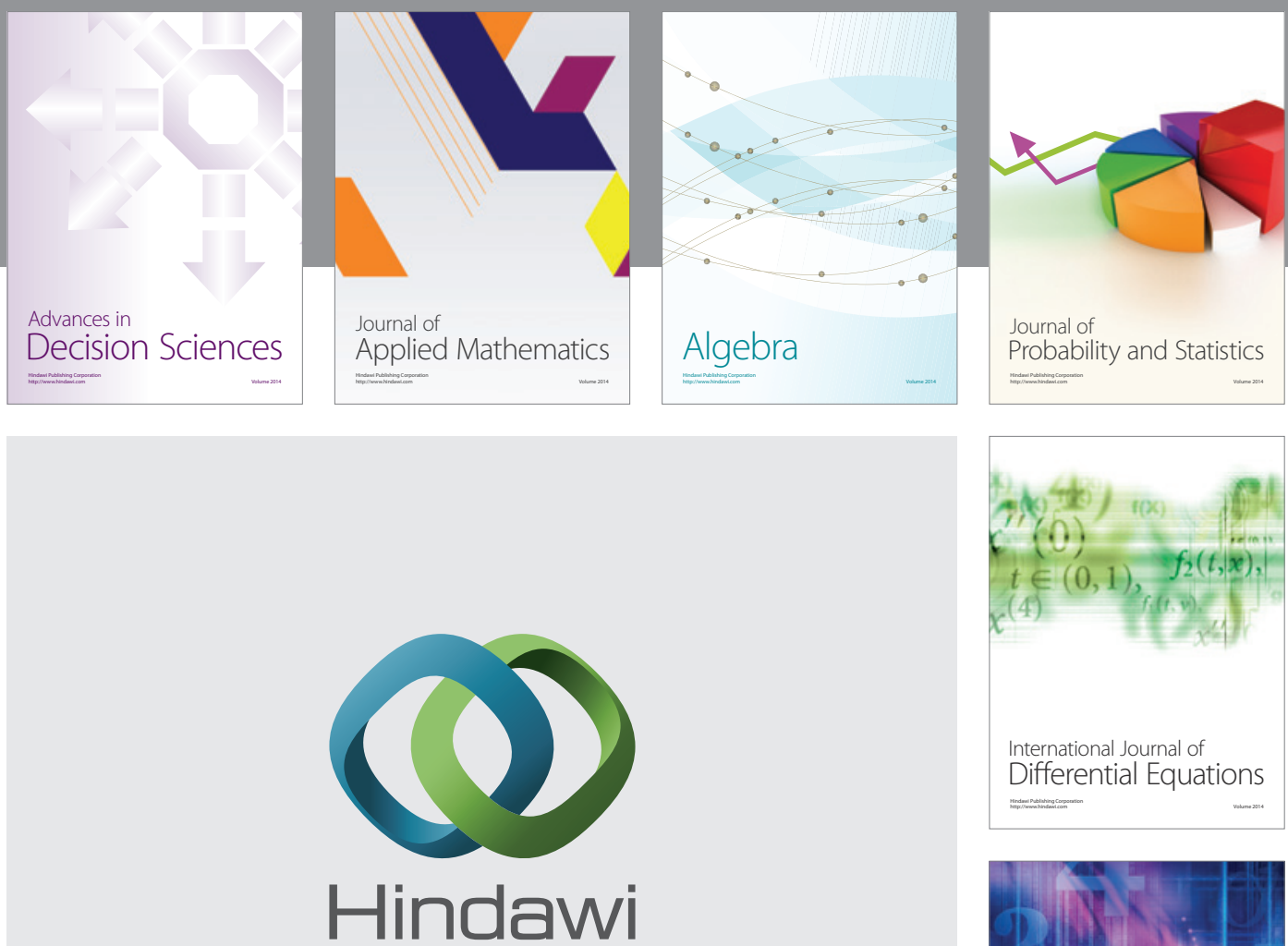

Submit your manuscripts at http://www.hindawi.com
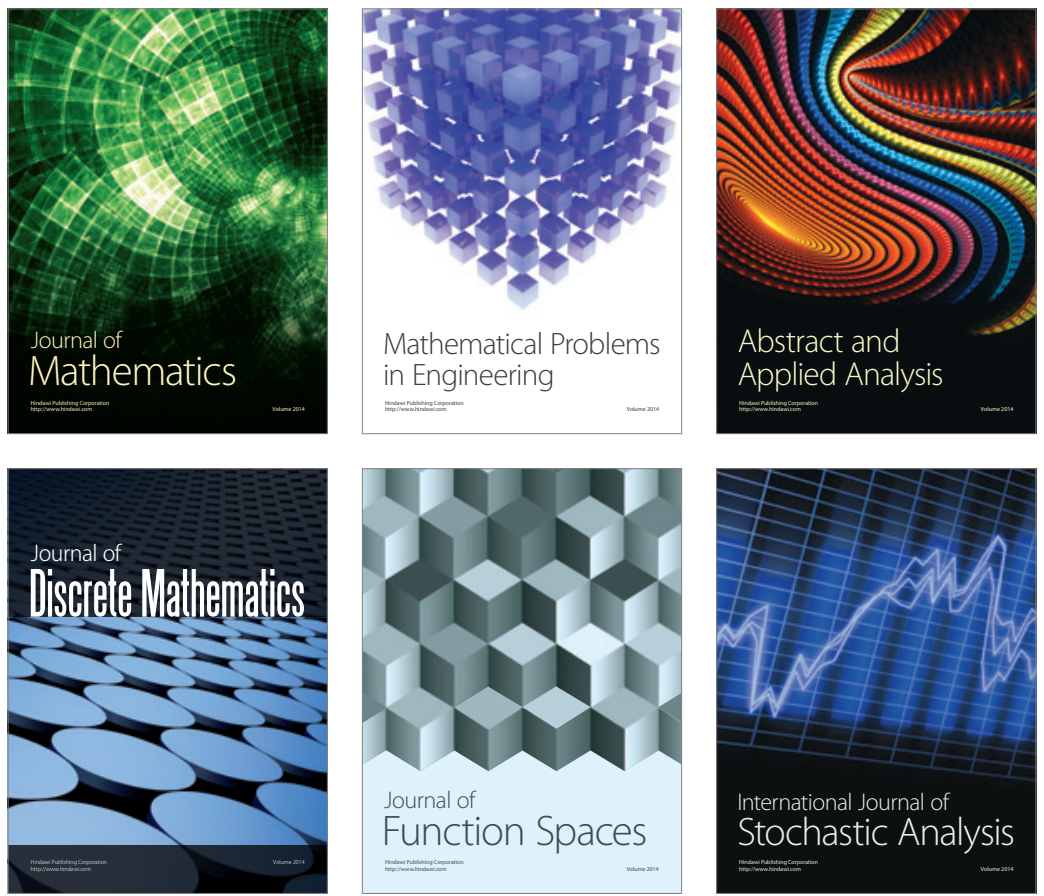

Journal of

Function Spaces

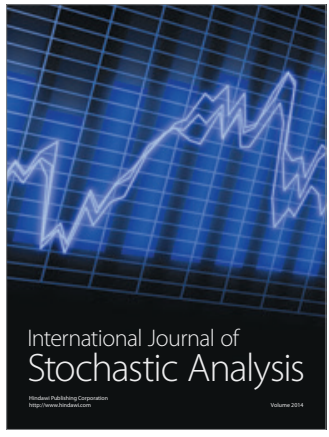

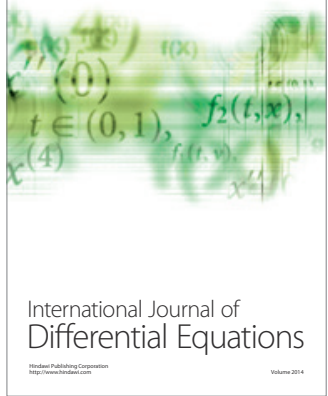
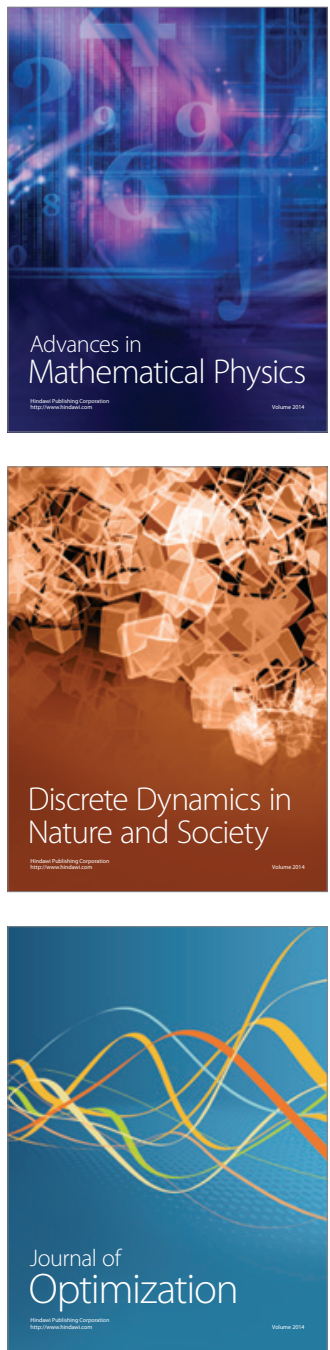